\title{
Hippocampal-medial prefrontal event segmentation and integration contribute to episodic memory formation
}

Wei Liu ${ }^{1}$, Yingjie Shi ${ }^{1}$, James N. Cousins ${ }^{1}$, Nils Kohn ${ }^{1}$, Guillén Fernández ${ }^{1}$

1. Donders Institute for Brain, Cognition and Behaviour, Radboud University Medical Centre,

Nijmegen, The Netherlands

\section{Correspondence:}

Wei Liu

Department of Cognitive Neuroscience

Donders Institute for Brain, Cognition and Behaviour

Radboud University Medical Centre

Trigon Building, Kapittelweg 29

6525 EN Nijmegen, The Netherlands

Tel: +31 (0)2436 53276

E-mail: W.Liu@donders.ru.nl 


\begin{abstract}
How do we encode our continuous life experiences for later retrieval? Theories of event segmentation and integration suggest that the hippocampus binds separately represented events into an ordered narrative. Using an open-access functional Magnetic Resonance Imaging (fMRI) movie watching-recall dataset, we quantified two types of neural similarities (i.e., activation pattern similarity and within-region voxel-based connectivity pattern similarity) between separate events during movie watching and related them to subsequent retrieval of events as well as retrieval of sequential order. We demonstrate that distinct activation patterns of the hippocampus and medial prefrontal cortex form event memories. By contrast, similar within-region connectivity patterns between events facilitate memory formation and are relevant for the retention of events in the correct sequential order. We applied the same approaches to an independent movie watching fMRI dataset and replicational analyses highlighted again the role of hippocampal activation pattern and connectivity pattern in memory formation. We propose that distinct activation patterns represent neural segmentation of events while similar connectivity patterns encode context information, and therefore integrate events into a narrative. Our results provide novel evidence for the role of hippocampal-medial prefrontal event segmentation and integration in episodic memory formation of real-life experience.
\end{abstract}

Keywords: subsequent memory effect; hippocampus; medial prefrontal cortex; event segmentation; event integration 


\section{MAIN TEXT}

\section{Introduction}

How we form memories of our life experiences is a fundamental scientific question with broad implications. In the past two decades, human neuroimaging and electrophysiology studies using the subsequent memory effect paradigm have implicated a distinct set of brain regions involved in successful memory formation (Brewer et al. 1998; Wagner et al. 1998; Fernández et al. 1999; Kim 2011). In these subsequent memory studies, increased neural activity of the hippocampus, parahippocampal gyrus, and the prefrontal cortex during memory encoding is associated with successful subsequent retrieval. However, real-world memories are formed based on a continuous stream of information rather than the sequentially presented, isolated items used in most subsequent memory studies (Kim 2011). Potentially, continuous sensory experience is segmented into distinct events (i.e., event segmentation) (Baldassano et al. 2017; Zacks 2020) that are then bound together into a coherent narrative, preserving their sequential relationships (i.e., event integration) (Griffiths and Fuentemilla 2020). To examine episodic memory formation of real-life-like experiences in humans, we analysed brain activity using functional Magnetic Resonance Imaging (fMRI) while participants were watching a movie. Based on subsequent memory recall, we aimed at identifying brain regions and neural representational processes underlying event segmentation and integration during episodic memory formation.

Thanks to recent advances in statistical analysis of ongoing neural activity (Hermans et al. 2011; Cohen et al. 2017; Xue 2018; Nastase et al. 2019), naturalistic stimuli (e.g., movie, spoken narratives, music) have been increasingly used in neuroscience (Hasson et al. 2004; Hermans et al. 2011; Huk et al. 2018; Sonkusare et al. 2019). This is especially valuable for memory research because naturalistic stimuli can greatly enhance the ecological validity of experimental studies (Hasson et al. 2008; Baldassano et al. 2017; Chen et al. 2017; Montchal et al. 2019). Hasson and colleagues first investigated memory formation with cinematographic stimuli and demonstrated that brain activity was more correlated among participants for later remembered than forgotten events (Hasson et al. 2008). While that study uncovered regions that 
encode continuous experiences, the nature of representations in those regions remained unclear, particularly with regard to how episodes are segmented into separate events and then integrated into a coherent sequence.

Event segmentation theory suggests that continuous experiences need to be segmented into discrete event representations, and thereafter they can be better understood and encoded (Zacks et al. 2001, 2007; Zacks 2020). Two recent studies provided novel perspectives into segmentation theory. Using Multi-Voxel Pattern Analysis (MVPA) and a movie watching-recall dataset, Chen and colleagues showed similar activation patterns of the same events across individuals and event-specific reinstatements of activation patterns between encoding and retrieval (Chen et al. 2017). Following this, Baldassano and colleagues demonstrated a nested processing hierarchy of events ('hierarchical memory system', (Hasson et al. 2015)) from fine-grained segmentation in early sensory regions to coarse segmentation in regions of the higherorder default-mode network (e.g., medial prefrontal cortex (mPFC) and posterior medial cortex (PMC)). Importantly, boundaries of long events at the top of the hierarchy matched with event boundaries annotated by human observers and were coupled to increased hippocampal activity (Baldassano et al. 2017). These results demonstrated that human brains spontaneously used different activation patterns to represent events during continuous movie watching, and how these activation patterns reactivated during recall. Also, it may suggest that regions such as $\mathrm{mPFC}, \mathrm{PMC}$, and hippocampus encode events at the same level that we consciously perceive boundaries between events. However, it remains unclear how exactly this event segmentation at the neural level relates to subsequent memory recall.

Event segmentation alone is not sufficient for episodic memory formation of continuous real-life experiences. Temporal context theory suggests that it is essential to integrate segmented events into a coherent narrative via time, meaning, or other abstract features (Howard et al. 2005; Howard and Eichenbaum 2013). Therefore, a non-exhaustive list of questions are: (1) what are the neural underpinnings of event integration during continuous memory formation, (2) does integration occur in the same brain regions as segmentation, and (3) how does integration relate to subsequent memory recall. A 
promising approach to answer these questions is to examine local connectivity patterns (also called multivoxel correlation structure), which may represent a brain signal that integrates events (Tambini and Davachi 2019). This method was derived from rodent electrophysiology (Qin et al. 1997; Kudrimoti et al. 1999; Lansink et al. 2008) and has been used in human fMRI studies (Tambini and Davachi 2013; Hermans et al. 2017) to quantify distributed memory representations in neuronal assemblies. Recently, Tambini and Davachi (Tambini and Davachi 2019) proposed that activation patterns are the representations of specific perceptual inputs (e.g., stimuli), while local connectivity patterns reflect particular encoding contexts or states. However, the different mnemonic functions of activity patterns and connectivity patterns have yet to be compared empirically within a single study. If local connectivity patterns represent encoding context, they may facilitate integration across events. Examination of connectivity patterns alongside activation patterns would help to characterise how the brain simultaneously performs event segmentation and integration.

Recently, a hippocampal neural code that simultaneously tracked subdivisions of a continuous experience (i.e., events) and their sequential relationship was described in rodents' CA1 region (Sun et al. 2020). This neural code could be a fundamental neural correlate by which episodic experience is integrated, but has yet to be revealed in humans. Hippocampal activity was found to increase at the boundaries between two events during continuous experience (Ben-Yakov and Dudai 2011; Ben-Yakov et al. 2013; DuBrow and Davachi 2013; Baldassano et al. 2017; Ben-Yakov and Henson 2018), but what these hippocampal signals represent in terms of event segmentation and integration is not clear. Theoretical models proposed that increased hippocampal signal may reflect a rapid shift in mental representations (e.g., temporal and/or contextual information of an event) (Ranganath and Ritchey 2012; DuBrow and Davachi 2016; DuBrow et al. 2017). Therefore, it can be regarded as the neural signature of event segmentation. Alternatively, this increase may link to the integration of episodic memories across event boundaries, as suggested by scalp electrocorticography (EEG) studies (Sols et al. 2017; Silva et al. 2019) and the event conjunction 
framework (Griffiths and Fuentemilla 2020). However, fMRI evidence for the role of hippocampal signals in integration across events is still limited.

The current study aimed to reveal the neural underpinnings of the two processes in question - event segmentation and event integration - during memory formation of naturalistic experiences. To that end, we first analyzed an existing dataset (Baldassano et al. 2017; Chen et al. 2017) where participants watched a movie while being scanned (Figure 1A) and afterwards were instructed to freely recall the story of the movie (Figure 1B). This design allowed us to associate different neural measures during episodic encoding with subsequent memory retrieval (Figure 1C-D). We extracted voxel-wise Blood Oxygenation Level Dependent (BOLD) time courses during movie watching (encoding) from six predefined regions-ofinterest (ROI) in the 'hierarchical memory system' (Hasson et al. 2015) including early auditory and visual areas, posterior medial cortex, medial prefrontal cortex, hippocampus, and posterior parahippocampal gyrus (Figure 2A; Figure S1). To probe the role of a broader set of regions in event segmentation and integration, we repeated all analyses in each region of a neocortical parcellation (Schaefer et al. 2018) (Figure 2B). We first examined the relationship between ROI-based activity time courses and subsequent memory recall and replicated the classical subsequent memory effects (i.e., greater activation for remembered compared to forgotten events) in regions including the hippocampus as well as the posterior parahippocampal gyrus (Figure S2-3, details in Supplementary Materials). To dissociate the two event processes, we used voxel-wise activity (Figure 2C) from each ROI to quantify the similarity between neural representations of events by two different multivariate methods (i.e., activation and connectivity patterns) (Figure 2D-E). Before linking neural pattern similarities with subsequent memory, we first compared between-event and within-event pattern similarities. We predicted that if our multivariate methods capture event representations, within-event pattern shifts should be smaller than between-event pattern shifts. Then we reasoned that if the neural representation (activation or connectivity pattern) shows a large transition (i.e., lower neural similarity value) between two adjacent events, and if this dissimilarity associates with better subsequent memory for events, then this 
bioRxiv preprint doi: https://doi.org/10.1101/2020.03.14.990002; this version posted December 12, 2020. The copyright holder for this preprint (which was not certified by peer review) is the author/funder, who has granted bioRxiv a license to display the preprint in perpetuity. It is made available under aCC-BY-ND 4.0 International license.

representation might be involved in event segmentation (Figure 2E). By contrast, if the neural representation remains stable (i.e., higher similarity) across two or more neighboring events, and this stability relates to event memory as well as retention of the correct order for those events (i.e., order memory), then this representation may underlie event integration (Figure 2F). The relationship between neural event processing (i.e., segmentation and event integration) and memory formation was further cross-validated in an independent movie watching-recall dataset (replication dataset) that used a different experimental protocol with alternative movie stimuli.

A

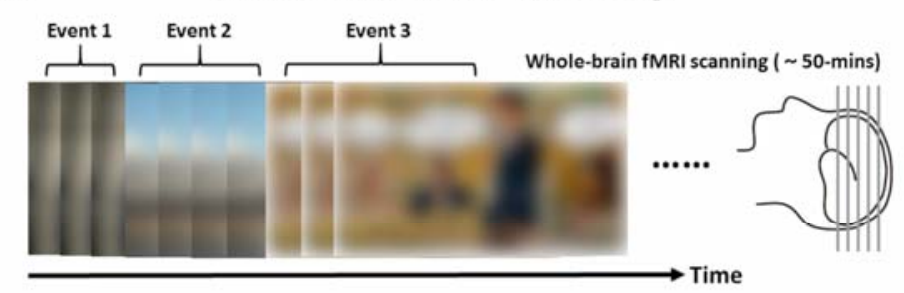

B

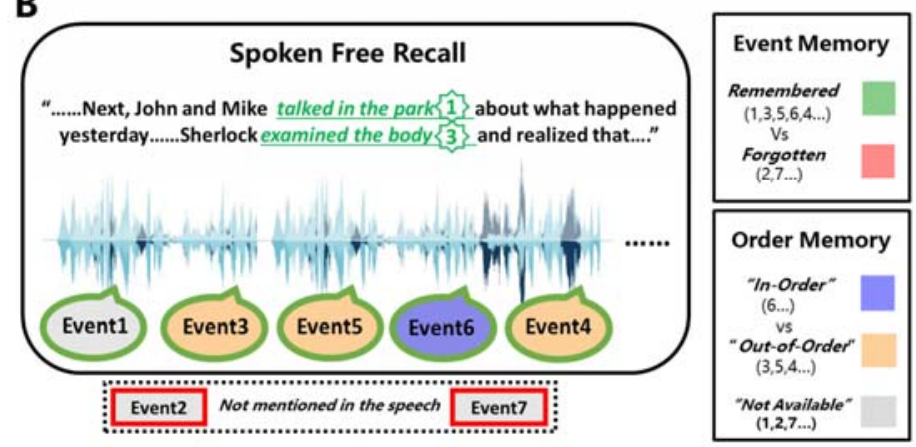

C Event Memory

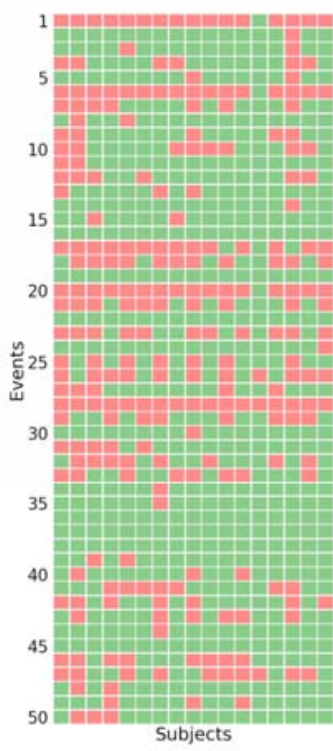

D Order Memory

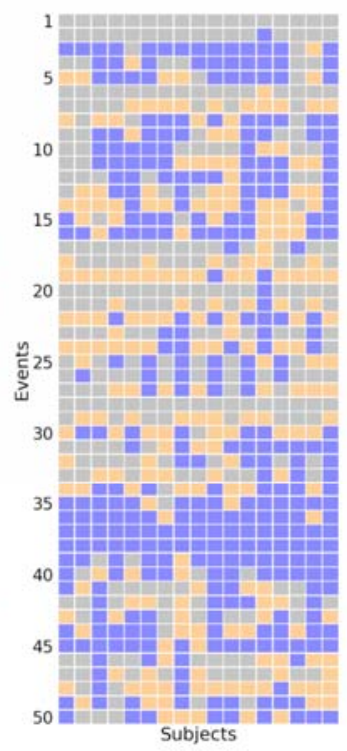

Figure 1. Experimental procedure and behavioural performance in the discovery dataset. (A) Each participant watched a 50-min audiovisual movie, BBC's Sherlock (season 1, episode 1), while brain activity was recorded with fMRI. The movie was divided into 50 events based on major narrative shifts. Blurred images are shown here due to copyright reasons. However, the movie was shown in high resolution during the experiment. (B) Immediately after movie-watching, participants verbally recalled the movie content in as much detail as possible without any visual or auditory cues. Speech was recorded using a microphone and then transcribed. Critically, speech was also segmented into events and matched with the events segmented from the movie. All events mentioned in the speech were labelled as remembered while missing events were labelled as forgotten. In addition, among those remembered events, the ones that were recalled in the correct sequential order were labelled as in-order events (e.g., event 6 was recalled after event 5). Out-of-order events were those that were recalled in an incorrect sequential order (e.g., event 4 was recalled after event 6 ). We labelled the first recalled event and all forgotten events as not available because no sequential information can be accessed. (C) Illustration of all remembered and forgotten events during movie-watching in all participants. (D) Illustration of all in-order and out-of-order events during movie watching in all participants. Each row of the heatmap is a different event, and each column represents a participant. 


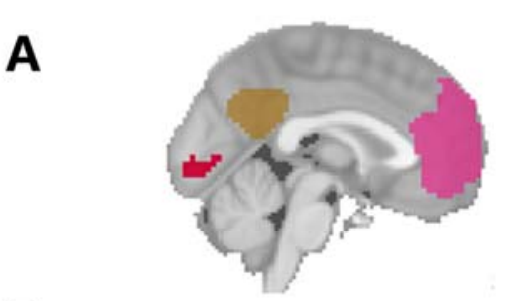

B
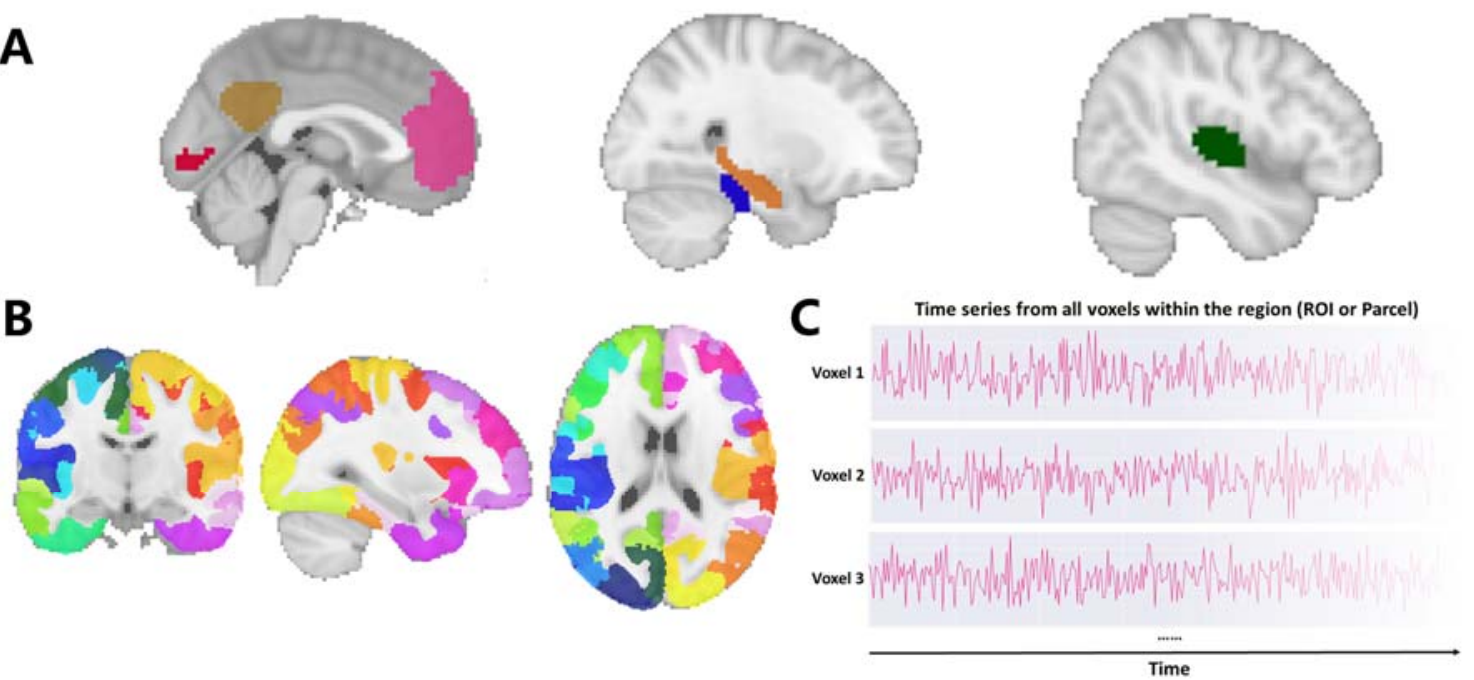

D
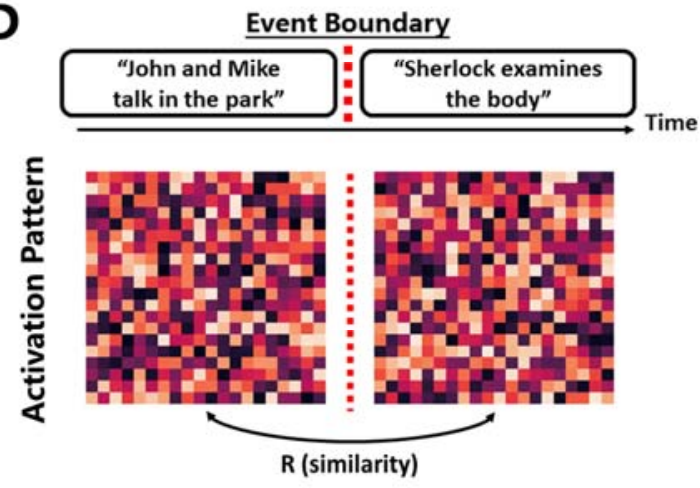

$\mathbf{F}$

Event Segmentation
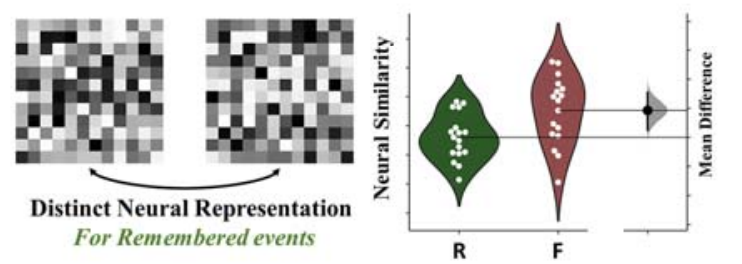

$\mathbf{E}$

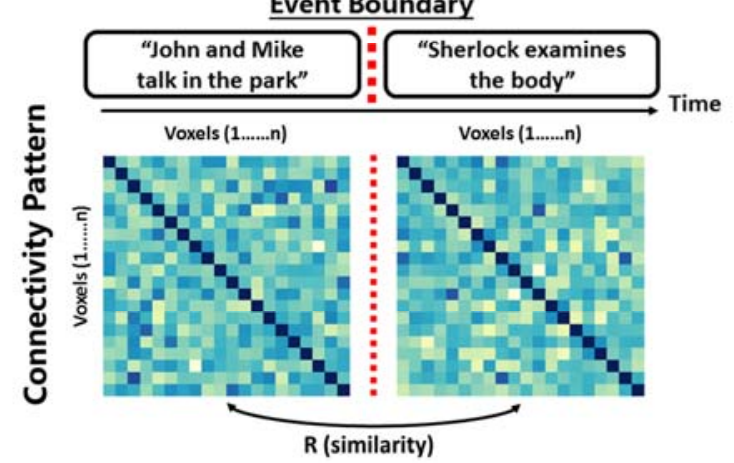

Event Integration
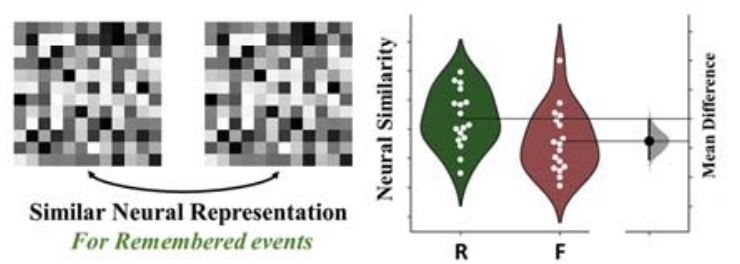

Figure 2. Neural similarities between separate events and their link with subsequent memory recall. (A) Six predefined regions-of-interest (ROIs): early auditory (green) and visual area (red), posterior medial cortex (brown), medial prefrontal cortex (pink), hippocampus (blue), and posterior parahippocampal gyrus (orange). See also Supplementary Figure 1. (B) Neocortical parcellation (1000 parcels) used in searchlight analysis. (C) For each region (ROI or parcel), voxel-wise signal during movie watching was extracted and then segmented into 50 events based on the event annotations. (D) We first generated event-specific activation patterns by averaging over all time points in that event. Then activation pattern similarity was calculated by Pearson's correlation between activation patterns of two sequential events. If a region encodes two events separately, we expect two distinct neural representations and therefore a lower pattern similarity. (E) Event-specific within-region connectivity patterns were represented by voxel-by-voxel pairwise correlation matrices. Connectivity pattern similarity across event boundaries was also calculated using Pearson's $r$ between two sequential events. Stable neural representations across two events should yield a higher pattern similarity in the corresponding region. (F) fMRI evidence for event segmentation. For a certain multivariate neural measure, if it can be found that two distinct neural representations are used to encode the adjacent events while the neural patterns for remembered (' $R$ ') events are more dissimilar compared to forgotten (' $F$ ') events, this measure is likely to be associated with event segmentation. (G) fMRI evidence for event integration. If the multivariate neural measure remains stable across the boundary of two neighboring events and remembered (' $R$ ') events have higher neural similarity compared to forgotten (' $F$ ') events, this measure may relate to event integration. 


\section{Methods}

\section{Participants and procedure}

\subsection{Participants}

\section{Discovery dataset}

Twenty-two healthy young adults (10 female, age range $18-26$, mean age 20.8 years) participated in the experiment. All participants were native English speakers and naïve to the BBC crime drama Sherlock. Data were discarded from participants with excessive motion $(>1$ voxel; $n=2)$, low recall duration $(<10$ $\min ; \mathrm{n}=2)$, or sleeping during the experiment $(\mathrm{n}=1)$. This leaves 17 participants in total for our analyses. Due to a technical problem, one participant (s5) is missing data for the last $75 \mathrm{~s}$ (part of event 49 and all of event 50) and the affected two events were excluded in the analyses.

\section{Replication dataset}

In total 52 healthy adults ( 40 older adults (mean age=69 years) and 12 young adults (mean age=23 years)) from the St. Louis community or Washington University's Psychology Department participant pool participated in this study. No participant reported current physical or mental health disorders. All of them were right-handed and had normal or corrected to normal vision. This research was approved by the Human Research Protection Office at Washington University

\subsection{Procedure}

\section{Discovery dataset}

All our analyses are based on the Sherlock Movie Dataset (Baldassano et al. 2017; Chen et al. 2017); see Data availability below) acquired and pre-processed at Princeton Neuroscience Institute. No similar analysis or results (excluding behavioural results of recall accuracy) have been reported in previous studies using this dataset. 
Participants were informed that they would watch a movie and would later be required to recall its content. They were then presented with a 48-min segment of the first episode of the Sherlock series (encoding phase), split into two parts of approximately equal length (23 min and $25 \mathrm{~min}$ ) and presented in two consecutive blocks. A $30 \mathrm{~s}$ introductory cartoon clip was prepended before each block. Immediately after the movie presentation, participants were instructed to verbally describe the movie in as much detail as they could and for as long as they wished (recall phase). They were asked to recall the episode in the correct sequential order but were permitted to return to earlier points in the narrative if they remembered further content. Audio was simultaneously recorded by a customized MR-compatible recording system throughout the recall phase.

\section{Replication dataset}

A detailed description of the procedure can be found in the previous publication (Kurby and Zacks 2018). Participants finished two sessions with an interval of around 3.4 days $(\mathrm{SD}=2.3$ days, $\min =0, \max =14$, mode=2). In session 1 (fMRI session), participants watched five movie clips in the same order. They were instructed to remember movie contents as much as possible. In session 2 (i.e., Behavioral session), two kinds of behavioral testing were performed. The first was the segmentation task, where participants were instructed to "press a button to press a button when, in their opinion, one meaningful unit of activity ended and another began" (Kurby and Zacks 2018). They produced both coarse (i.e., largest meaningful units) and fine (i.e., smallest meaningful units) segmentation for the same clip and we used the coarse segmentation as event boundaries in our neuroimaging analyses. Second, they performed memory tests. Recognition, recall, and order memory was tested for each movie clip. Recognition memory was tested using a 20-item two-alternative forced-choice test where participants were instructed to choose the visual image they saw in the movie instead of the distracter. Recall memory was assessed by asking participants to describe the movie content in as much detail as possible. Order memory was tested by reordering 12 visually distinctive images from the movie according to when they appeared. 


\section{Behavioural data analysis}

\section{Discovery dataset}

\section{Event annotations of the movie and verbal speech recording}

The movie was segmented into 48 events by an independent observer who was blind to the experimental purpose, design, or results, following major shifts in the narrative (e.g., changes in location, topic, and/or time). Each event was given a descriptive label (e.g., "press conference"). Including the two introductory cartoon clips, 50 scenes were analysed in total. The timestamps for both the onset and offset of identified scenes were recorded and aligned across all participants. Both the onset and offset are referred to as the boundaries of the respective event. This is a widely used method for event segmentation and has been validated by a data-driven approach (Baldassano et al., 2017). The length of the events ranges from 11 to 180s (Mean \pm SD: $57.5 \pm 41.7$ s). The distribution of event length is visualized in Figure S1, and the duration of each event is presented in Table S1. Each subject's verbal speech was transcribed, segmented, and matched to the events that were recalled from the movie.

\section{Situational variables of movie events}

For each movie event, several situational variables including both semantic (e.g., location) and affective features (e.g. arousal) were analyzed together with the subsequent recall of that event in both correlational analyses and mixed-effect modeling (see validation analysis below). Firstly, the entire movie was divided into 1000 time segments (mean duration=3.0s, s.d.=2.2s) by a human rater. Each of the 1000 segments was then labeled for variables including arousal (excitement/engagement/activity level), music (whether or not there is music playing), location (whether the location is indoor or outdoor), and valence (Positive or negative mood). For subjective rating (i.e., arousal and valence), assessments were collected from four different raters (arousal: Cronbach's $\alpha=0.75$; valence: Cronbach's $\alpha=0.81$ ). In the event-specific analyses, a score was derived for each of the 50 events for each of the variables mentioned by averaging/adding up ratings across time segments. The event-level scores are displayed in Table S1. These 
analyses were performed and data were shared by Chen and colleagues (Chen et al. 2017). We correlated these variables (along with event duration) with the mean recall rate in all participants (Figure S5) and found that arousal and event duration positively correlated with memory recall $(\mathrm{p}<0.05)$. Other situational variables did not show significant correlations with memory $(\mathrm{p}>0.05)$.

\section{Event and order memory}

For each participant, we first asked whether events were successfully recalled or not, as in the classical subsequent memory paradigm (Brewer et al. 1998; Wagner et al. 1998; Fernández et al. 1999). An event was labeled as 'remembered' if any part of the event was described during the recall. 'Forgotten' events are the ones that were not mentioned throughout the recall phase.

Secondly, out-of-order events were identified as a measure of order memory. Among all remembered events, an event was labelled as out-of-order if it was not described immediately after its preceding event in the original movie. For example, if event 3 is described immediately after event 1 without mentioning event 2, then event 3 is an out-of-order event. By contrast, if a participant described event 4, 5, 6 sequentially during the recall phase, since event 5, 6 correctly followed their preceding event, event 5, 6 were counted as in-order events. The first event verbally described in the recall phase was always labelled as 'not available' in the order memory analysis since it is not preceded by any event. It was possible that a single scene was mentioned multiple times (in different parts) during the recall, in which case the position of its first recall was used in the event and order memory analyses.

\section{Replication dataset}

All of the behavioral results were performed and shared by authors (C.K and J.Z) of the original publication (Kurby and Zacks 2018). Coarse event boundaries for our neural pattern similarity analyses.

Recognition memory was measured as the percent of correct responses during the forced-choice test.

Action recall memory was scored by how many of each type of action was mentioned (e.g., man drinking coffee). This scoring procedure was based on the Action Coding System (ACS: (Schwartz et al. 1991)). 
Raw order memory was assessed as the number of errors made in the reordering test. To generate the (final) order memory measure, we multiplied each raw order memory measure by -1 and Z-normalized across all participants. This transformation made the measure intuitive (i.e., larger values indicate better order memory).

\section{3. fMRI data analysis}

\section{1 fMRI data acquisition and pre-processing}

\section{Discovery dataset}

fMRI data were acquired using a T2*-weighted EPI sequence on a 3T Siemens Skyra scanner (20-channel head coil; TR 1,500 ms; TE $28 \mathrm{~ms}$; flip angle 64 , spatial resolution $3 * 3 * 4 \mathrm{~mm}^{3}$ ). Only data from the encoding phase were analysed and reported in the current study.

A standard pre-processing pipeline was followed using FSL (Jenkinson et al. 2012), which includes slice timing correction, motion correction, linear detrending, high-pass filtering (140 s cutoff), co-registration and affine transformation into $3 \mathrm{~mm}$ MNI standard space (Chen et al. 2017). The time series were shifted 3 TRs (4.5 s) to account for the Haemodynamic response function (HRF). Data were z-scored across time at every voxel and a $6 \mathrm{~mm}$ smoothing kernel was applied.

All subsequent analyses were performed on the pre-processed voxel-wise BOLD signal, in units of functional volume (TR = $1.5 \mathrm{~s})$. Custom MatLab (R2018b, The Mathworks, Natick, MA) and Python (version 3.6) scripts were used for both Region of Interest and parcellation-based searchlight analysis.

\section{Replication dataset}

Neuroimaging data were acquired with a Siemens Trio 3T scanner. Functional data (i.e., movie watching data) were acquired in five runs using a T2* weighted EPI sequence (TR $=2000 \mathrm{~ms}$, TE $=27 \mathrm{~ms})$ in 35 transverse slices (voxel size $=4.0 \mathrm{~mm}$ ). We re-ran the preprocessing according to the pipeline used in the 
discovery dataset. Subsequent neuroimaging data analyses were performed on the preprocessed voxel-vise BOLD signal for each ROI.

\subsection{Region of interest (ROI) selection}

The six ROIs used in this study were independently defined by Chen and colleagues, in correspondence to the timescale hierarchy of the event segmentation model (Hasson et al. 2015; Baldassano et al. 2017). Early visual and early auditory cortex were functionally defined based on inter-subject correlation during an audio-visual movie and an audio narrative, respectively (Chen et al. 2016; Simony et al. 2016). ROIs for medial prefrontal cortex (mPFC) and posterior medial cortex (PMC) were taken from the functional atlas derived from resting-state default mode network (https://findlab.stanford.edu/functional_ROIs.html) from FIND lab at Stanford University (Shirer et al. 2012). The hippocampus and posterior parahippocampal gyrus were anatomically defined from the probabilistic Harvard-Oxford Subcortical Structural Atlas (Desikan et al. 2006). Chen and colleagues manually adjust the threshold of around 50\% to ensure better anatomical coverage during the visual check.

\subsection{Whole-brain parcellation}

Alongside the ROI-based analysis, we performed a parcel-based searchlight analysis on the basis of 1000 functionally parcellated cerebral regions (https://github.com/ThomasYeoLab/CBIG/tree/master/stable_projects/brain_parcellation/Schaefer2018_L ocalGlobal). The parcellation was based on a gradient-weighted Markov Random Field (gwMRF) model, which integrated local gradient and global similarity approaches (Schaefer et al., 2018). Using both task and resting-state fMRI acquired from 1489 participants, parcels with functional and connectional homogeneity within cerebral cortex were generated (hippocampus and subcortical regions were not included). In this fashion, each of these biologically meaningful and non-overlapping parcels can be treated in the same way as an independent region similar to an ROI in the following analyses. The 
parcellation was provided in both volume and surface space, and the volume-based parcellation space was used in our searchlight analyses.

\section{4 fMRI-based neural responses to event boundaries}

\subsubsection{Univariate response}

BOLD signals were first averaged for each TR across all voxels in an ROI. Then the time series were zscored and segmented based on the event annotations mentioned above. We selected the time window of the univariate response analysis based on the shortest event duration. Among all events, the shortest event was 7 volumes $(10.5 \mathrm{~s})$, therefore we focused on BOLD signals 6 volumes before and after the event boundaries (i.e., in total 13 volumes around event boundaries).

\subsubsection{Activation patterns}

Voxel-wise BOLD time series from separate events were first extracted based on the onset and offset timestamps derived from the movie. Multivariate patterns of brain activation were generated for each event by averaging across all volumes within this event. To assess the similarity between two neighboring events, the activation pattern for each event of interest was correlated with its following event. The resulting Pearson's correlation coefficient depicted the extent to which similar representational activity patterns were elicited by neighboring scenes. Lower similarity between two events represented a greater change in neural patterns across the event boundary.

\subsubsection{Connectivity patterns}

Intra-regional connectivity pattern analyses were conducted based on a method originally used in rodent electrophysiology studies to quantify the reactivation of sparsely distributed neuron assemblies (Qin et al. 1997; Lansink et al. 2008), and recently used in human fMRI (Tambini and Davachi 2013, 2019; Hermans et al. 2017). For each event within each brain region, Pearson's correlations were performed on the extracted $m^{*} n$ (volumes*voxels) BOLD-fMRI time series, between each of the $\mathrm{n}$ voxel time series. This 
yielded an n-by-n pairwise correlation matrix (containing $\mathrm{p}$ values indicating the significance of the Pearson's correlations), representing the within-region connectivity structure for each scene. For two neighboring events, the Pearson's correlation coefficient of their correlation matrices was calculated to quantify the similarity for connectivity patterns. Lower similarity between two connectivity patterns represented a greater change in the intra-region connectivity patterns across the event boundary.

\subsubsection{Activation/Connectivity pattern similarity within and between movie events}

We compared within-event and between-event activation/connectivity pattern similarities to reveal the effects of event boundaries on neural pattern shifts. First, we generated "middle-point boundaries" for within-event neural similarities calculation. Specifically, for each of the fifty original events, an additional middle-point boundary was located at the middle points of the corresponding time series. Thus, one original event can be divided into two "half events" with equal duration. This created 100 "half events" defined by both human-annotated boundaries and "middle-point boundaries". Then, we quantified neural similarities of activation/connectivity patterns between two neighbouring "half events". If two "half events" were segmented by a human-annotated boundary, then the similarity was defined as the "between-event" pattern similarity, whereas if the two "half events" were segmented by a middle-point boundary, the similarity was defined as the "within-event" pattern similarity.

\subsection{Relationship between neural responses during encoding and subsequent memory}

\section{Discovery dataset}

\subsubsection{Remembered and forgotten events comparisons}

We first compared our neural pattern similarities (i.e., activation pattern similarity and connectivity pattern similarity) at the single-subject level explained above for each brain region (ROI or brain parcel). The similarity indices (Pearson's $r$ between two matrices) for both activation and connectivity patterns were averaged for the two types of event pairs (remembered and forgotten) for each participant. If the first event of the pair was retrieved during the recall phase, the event pair was labeled as remembered. 
Remembered and forgotten event pairs were then compared in two separate $t$-tests for activity and connectivity pattern transitions (indexed by pattern similarity).

We further examined the relationship between connectivity pattern transitions and order memory (i.e., temporal order of event recall). More specifically, connectivity patterns were averaged for another two types of event pairs (i.e., In-order or Out-of-order) for each participant. If the second event of the pair was recalled in an incorrect sequential order (e.g., event 4 was recalled immediately after event 6 ), the event pair was labeled as Out-of-order. Connectivity pattern transitions for In-order and Out-of-order event pairs were then compared with $t$-tests.

\subsubsection{Validation analyses of subsequent memory effects}

Beyond the paired t-tests between neural similarities of Remembered and Forgotten events, in total six additional statistical tests were performed to further validate reported subsequent memory effects (Detailed methods and results can be found in Supplementary Materials). In brief, (1) neighboring event pairs were divided into four categories based on memory for both the first and second event of the event pair (i.e., both Forgotten (FF), first Forgotten and second Remembered (FR), first remembered and second forgotten $(R F)$, and both remembered $(R R))$. We then compared neural similarities across these four categories. (2) To confirm that the subsequent memory effect on pattern similarity was only present for actual event boundaries but not shuffled boundaries, we generated a null distribution of subsequent memory effects using the event boundary permutation analysis. During each permutation, event boundaries were re-located within events to create the same number of pseudo-events. The sequence of memory labels remain unchanged, and subsequent memory effects were qualified based on pseudo-events and corresponding memory labels. We asked whether subsequent memory effects based on the actual boundaries were larger than effects based on shuffled boundaries. (3) Memory labels (i.e., $R$ and $F$ ) were shuffled across events, then neural pattern similarities were compared between shuffled memory labels instead of real labels. (4) For each event, we examined whether the likelihood of an event being remembered among all participants correlated with its mean neural pattern similarity with the previous 
event. (5) We also performed a cross-participant correlation: we asked whether participants who demonstrated better memory showed lower/higher activation/connectivity pattern similarity during movie watching. (6) We further used a mixed-effects model to examine the relationship between neural similarity and memory, considering both participants and events as random effects and incorporating multiple eventspecific situational variables (e.g., event duration, location, music, arousal...).

\section{Replication dataset}

Subsequent memory for each movie clip was assessed by three different memory measures (i.e., recognition, recall, and order memory). Their relationships with event processing during encoding were investigated as a conceptual replication of findings from the discovery dataset. We calculated neural pattern similarities using the "within-movie" and "between-movie" method separately. For the "withinmovie" method, participant-specific boundaries generated during the segmentation task were used as event boundaries to calculate event-specific fMRI activation patterns and connectivity patterns during movie watching. Similarities of these activation/connectivity patterns were calculated across boundaries and then averaged for each movie clip at the participant level. To probe the memory relevances of these mean similarities, they were correlated with all three memory measures. For the connectivity pattern similarity analyses, we additionally used the "between-movie" method to enable meaningful connectivity analyses with enough TRs. Each movie clip was regarded as an event, and the connectivity pattern was estimated within the entire clip. Then connectivity pattern similarities were calculated across different movie clips and correlated with memory measures.

\subsection{Relationship between hippocampal pattern similarity and event distance}

The above analyses focused on neural pattern similarities between two neighboring events. Here, we examined the hippocampal pattern similarities between events with variable distances. Event distance was defined as the number of event boundaries between two events (the event distance between event 1 and event 3 is 2). For each event, we first calculated its activation and connectivity pattern. Then, we 
calculated the activation and connectivity pattern similarity between all possible combinations of event AB pairs ('Event A' is the event which appeared earlier in the temporal sequence, and 'Event B' is the one presented later) within all 50 events. Finally, for each participant, and each event distance, two mean similarities for activation and connectivity pattern were calculated separately. Note that the number of available pairs decreases as the distance increases (e.g., events 1-50 are the only event pair with a distance of 49). To ensure a well-powered analysis for every event distance, we only compared event pairs with a distance less than or equal to 40 , meaning at least 10 event pairs contributed to the event distance calculation. Analysis of all distances $(\mathrm{d} \leq 49)$ can be found in the Supplementary Materials.

Next, we used linear regression to examine the relationship between pattern similarity and event distance. In addition, to investigate how the subsequent memory of the preceding event (event A) modulates the relationship between event distance and pattern similarity, we ran a two-way ANOVA (memory * event distance) using the memory performance (remembered or forgotten) of the preceding event and event distance (range from 1 to 40) as two independent variables. The relationship between memory and event distance was validated with the permutation test, in which memory labels (i.e., $\mathrm{R}$ and F) were shuffled randomly 1000 times to generate null comparisons between two kinds of events.

\section{Statistical analysis}

For parametric hypothesis tests involved in the fMRI data analyses, the significance level was set to $p=$ 0.05 (two-tailed). For permutation tests, $p$-values were estimated by comparing real results with null distributions generated by shuffling event boundaries or memory labels, and their significance levels were also set to $p=0.05$ (two-tailed). To account for the multiple comparisons problem that comes with multiple ROIs or brain regions, all reported $p$ values in the main text were FDR-corrected $\left(p_{\text {FDR }}\right)$ (Genovese et al. 2002) unless otherwise stated $\left(p_{\text {raw }}\right)$. Specifically, this means correction was made for six tests in ROI analyses, and 1000 tests for the whole-brain analyses. All significant $p$ values were reported together with the effect sizes (Cohen's d or partial $\eta^{2}$ ). The custom modified version of DABEST 
(https://github.com/ACCLAB/DABEST-python) was used to plot individual data points alongside otstrapping-based resampled distributions of the mean difference between conditions (Ho et al. 2019).

\section{Data and code availability}

ROI data are available at http://datasets.datalad.org/?dir =/workshops/mind-2017/sherlock. Whole-brain neuroimaging data are available at https://dataspace.princeton.edu/jspui/handle/88435/dsp01nz8062179. The replication dataset was stored at The Central Neuroimaging Data Archive (CNDA), Washington University, Saint Louis (https://cnda.wustl.edu/) and can be requested from Dynamic Cognition Laboratory (https://dcl.wustl.edu/people/jzacks/). Custom code used in this study will be publicly available via the Open Science Framework (OSF) (Link: https://osf.io/p68cv/?view_only=483703873dae4cfd8b36e9d6df6b8c92) upon publication. Further requests for scripts should be directed to the corresponding author.

\section{Results}

\section{Subsequent memory performance measured by spoken recall}

We first calculated recall accuracies for each participant. On average, $68.7 \%(S D=12 \%$, range $48 \%$ 94\%) of the 50 events (Mean $=34.4$ events, $S D=6)$ were retrieved successfully (Figure 1C). Among these remembered events, we further defined in-order and out-of-order events based on whether they were recalled in the correct sequential order. On average, $58.8 \%(S D=8 \%$, range $40 \%-71 \%)$ of the remembered events were in-order (Figure 1D).

\section{Neural pattern shifts were larger for between-event transitions compared to within-event transitions}

Before linking neural pattern shifts to subsequent memory recall, we investigated how event boundaries modulated neural pattern shifts of both activation and connectivity patterns. More specifically, we compared the effect of between-event transitions compared to within-event transitions on neural pattern 
shifts (Figure S6A). Paired t-tests between within-event and between-event similarities revealed that in all six ROIs: (1) within-event activation similarities were significantly higher than between-event activation similarities; (2) higher connectivity pattern similarities were found for within-event transitions compared to between-event transitions (Figure S6B; Table S2). These results suggest that neural patterns are relatively stable within each event, but shift significantly across events.

\section{Distinct activation pattern-mediated event segmentation is associated with subsequent retrieval}

\section{success}

We quantified neural similarities of event-specific activation patterns before and after event boundaries (i.e., two neighbouring events). Specifically, we generated a voxel-wise activation pattern per event by averaging over all time points in that event. This time-averaged activation pattern of all voxels within an ROI for an event was compared to the pattern of its subsequent event using Pearson's correlation. A lower Pearson's $r$ indicates two more separateble activation patterns and thus more distinct neural representations for two distinct events. We investigated whether activation pattern similarities relate to memory formation by contrasting the pattern similarities of remembered with forgotten events in six ROIs. That is, pattern similarity between two events was compared to subsequent memory for the first of those events. We found that subsequently remembered events were associated with lower activation pattern similarities than subsequently forgotten events in early auditory cortex $\left(t=-3.56, p_{\mathrm{FDR}}=0.007\right.$, Cohen's $\mathrm{d}$ $=0.92$, Figure 3B $)$, hippocampus $\left(t=-3.62, p_{\mathrm{FDR}}=0.007\right.$, Cohen's $\mathrm{d}=0.92$, Figure 3E $), \mathrm{mPFC}(t=-2.79$, $p_{\mathrm{FDR}}=0.01$, Cohen's $\mathrm{d}=0.80$, Figure 3C) and posterior parahippocampal gyrus $(\mathrm{pPHG})\left(t=-2.85, p_{\mathrm{FDR}}=\right.$ 0.01, Cohen's d $=0.89$, Figure 3F). This finding suggests that distinct activation patterns for two sequential events are beneficial for the memory of the first event in that sequence. Early visual areas $(t=-$ $1.13, p_{\mathrm{FDR}}=0.27$, Cohen's $\mathrm{d}=0.35$, Figure 3A $)$ and $\mathrm{PMC}\left(t=-1.91, p_{\mathrm{FDR}}=0.08\right.$, Cohen's $\mathrm{d}=0.65$, Figure 3D) did not show this marked effect.

Beyond the main contrasts above (i.e., paired t-tests) between activation pattern similarity of remembered and forgotten events, we ran several additional statistical tests to further examine the relationship between 
activation pattern similarity and memory. Detailed methods and results from each ROI can be found in the Supplementary Materials.

(1) In our main analyses above, we labeled an event pair as "remembered" if the first event of the pair was remembered. In control analyses, we probed the potential effects of the second event and/or the interaction between the first and second event: event pairs were divided into four categories based on memory (i.e., both Forgotten (FF), first Forgotten and second Remembered (FR), first remembered and second forgotten $(R F)$, and both remembered $(R R))$ ), and compared neural similarities across these four categories. Consistent with main contrasts, hippocampal activation pattern similarities tended to be lower for RR pairs compared to FF pairs $\left(\mathrm{t}=-1.89, p_{\text {raw }}=0.07\right)$. Significant effects were found for the early auditory area $(\mathrm{t}=-$ $\left.2.32, p_{\text {raw }}=0.03\right), \operatorname{mPFC}\left(\mathrm{t}=-3.32, p_{\text {raw }}=0.005\right)$, and $\mathrm{pPHG}\left(\mathrm{t}=-3.36, p_{\text {raw }}=0.004\right)$. Full comparations of four categories can be found in the Figure $\mathbf{S 7}$.

(2) To test whether presented results only existed for the actual event structure, we generated shuffled event boundaries and re-ran the same contrasts on activation pattern similarity. Permutation tests demonstrated that presented subsequent memory effects only existed for the actual event boundaries, but not shuffled boundaries (Figure S8).

(3) The percentage of remembered events was higher than forgotten events, leading to the potential power issue when comparing the two. To counter this, we evaluated the current statistical results by performing a second kind of permutation test: for each permutation, memory labels were shuffled across events, then activation pattern similarities were compared between shuffled memory labels instead of real labels. All ROIs showed inseparable distribution between shuffled $\mathrm{R}$ and $\mathrm{F}$ events in terms of (mean) activation pattern similarity values, while similarity values of real $\mathrm{R}$ and $\mathrm{F}$ events differed in an ROI-specific manner as reported (Figure $\mathbf{S 9 )}$.

(4) So far, within-participant comparisons between remembered and forgotten events revealed that differences in activation pattern similarities of several ROIs were related to subsequent memory. Next, we 
examined whether a similar relationship was evident across different events. Specifically, we investigated the relationship between the event-specific recall rate (the percentage of participants that successfully recalled a particular event) and the averaged activation pattern similarity for the corresponding event (the first one in the pair) across all participants. Consistent with our main contrasts, this analysis revealed that the recall rate negatively correlated with activation pattern similarity in the hippocampus $\left(\mathrm{r}=-0.292, p_{\text {raw }}\right.$ $=0.042)$ and $\mathrm{pPHG}\left(\mathrm{r}=-0.344, p_{\text {raw }}=0.015\right)$, suggesting that events showing lower activation pattern similarity with the subsequent event were more likely to be recalled (Figure S10).

(5) We further performed cross-participant individual differences analysis between activation pattern similarity and memory, but found no significant associations. There was a trend for those participants with average lower activation pattern similarity in the early auditory $\left(\mathrm{r}=-0.37, p_{\text {raw }}=0.13\right)$ and pPHG $(\mathrm{r}=-0.40$, $p_{\text {raw }}=0.11$ ) during movie watching performed better at the memory test (Figure S11; Table S3), which is consistent with results from our main contrast analyses.

(6) Finally, we used a mixed-effects model for statistical analysis to examine the relationship between activation pattern similarity and memory, considering both participants and events as random effects. The relationship between memory and hippocampal activation pattern similarity $\left(\mathrm{F}=3.48, p_{\text {raw }}=0.06, \mathrm{R}^{2}=0.004\right)$ failed to reach significance but demonstrated the same tendency as results from the paired t-test. In a second model, event-specific situational variables (e.g., event duration, location, music...) were further modeled as fixed effects to be controlled as confounds. Again, hippocampal activation pattern similarity $\left(\mathrm{F}=2.80, p_{\text {raw }}=0.09\right)$ showed the same tendency of memory effects but failed to reach significance (Table S4). 
bioRxiv preprint doi: https://doi.org/10.1101/2020.03.14.990002; this version posted December 12, 2020. The copyright holder for this preprint (which was not certified by peer review) is the author/funder, who has granted bioRxiv a license to display the preprint in perpetuity. It is made available under aCC-BY-ND 4.0 International license.

A

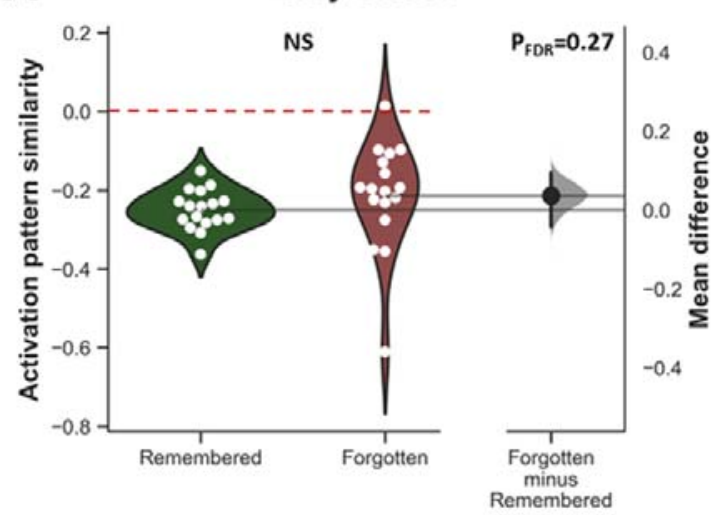

C

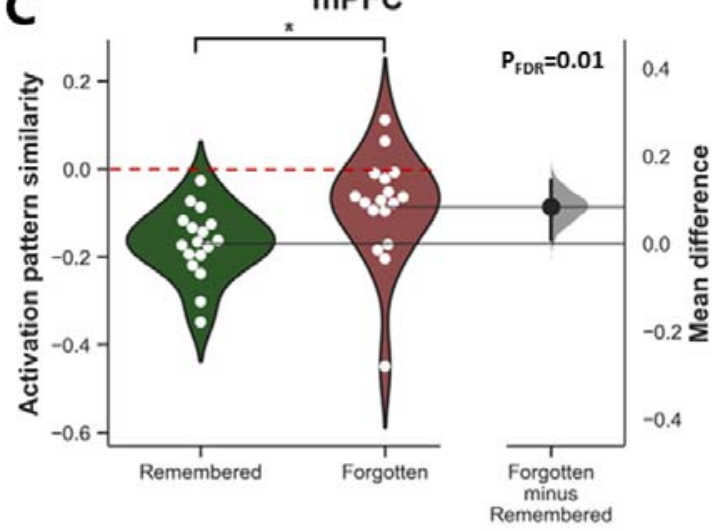

$\mathbf{E}$

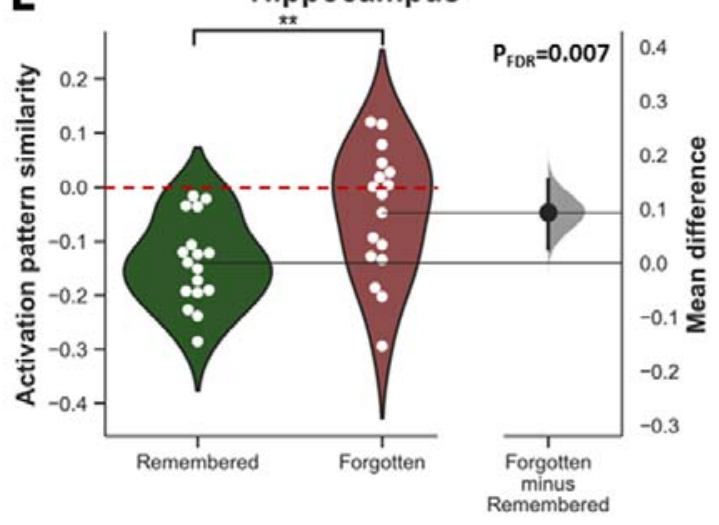

B

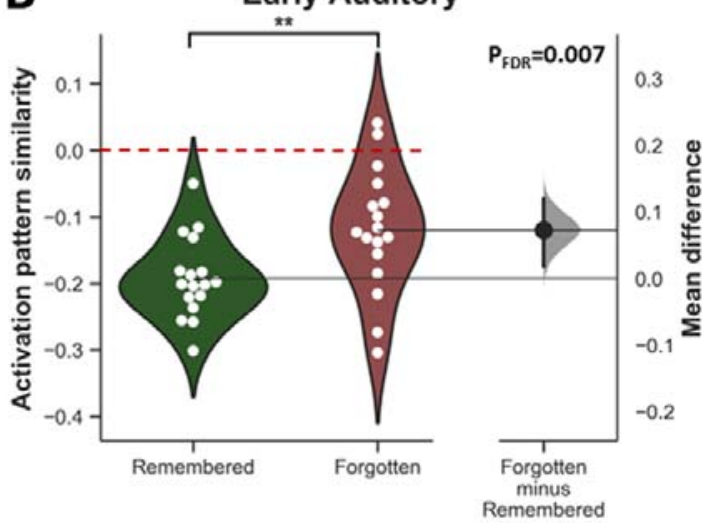

D

PMC

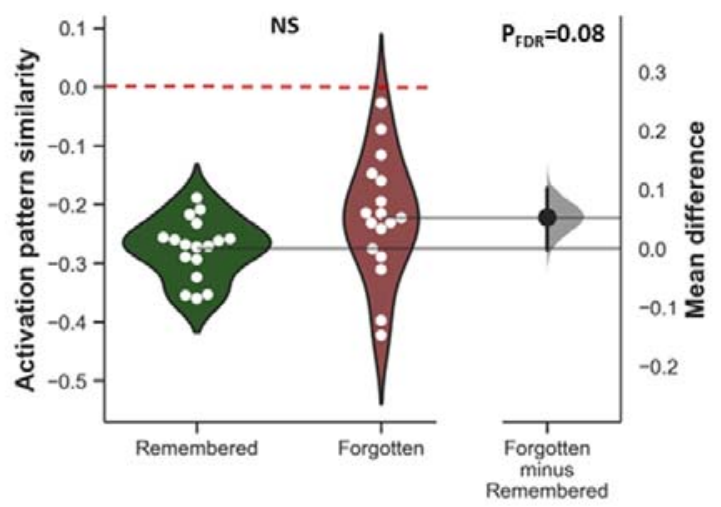

$\mathbf{F}$

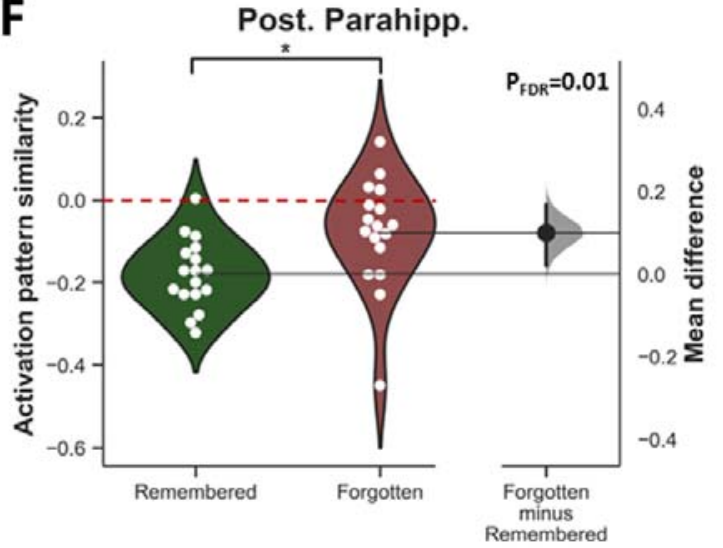

Figure 3. Association between activation pattern similarities of six ROIs and subsequent memory recall. We compared activation pattern similarities of sequential event pairs based on subsequent memory performance of the first event (Remembered vs. Forgotten) across six ROIs. For panel A-F, activation pattern similarities for Remembered events are displayed on the left (green), while similarities for Forgotten events are displayed on the right (red). For each comparison, a separate axis displays the mean difference. The curve (gray) indicates the resampled distribution of the mean difference generated via bootstrapping. The solid vertical line attached to the curve represents the mean difference as a 95\% bootstrap confidence interval. We found significantly lower activation pattern similarity for Remembered vs. Forgotten event pairs in the early auditory area $(t=-3.56$, $p_{\mathrm{FDR}}=0.007$, Cohen's d $=0.92$; panel B $), \operatorname{mPFC}\left(t=-2.79, p_{\mathrm{FDR}}=0.01\right.$, Cohen's $\mathrm{d}=0.80$; panel C $)$, hippocampus $\left(t=-3.62, p_{\mathrm{FDR}}\right.$ $=0.007$, Cohen's d $=0.92$; panel $\mathbf{E})$, and pPHG $\left(t=-2.85, p_{\mathrm{FDR}}=0.01\right.$, Cohen's d =0.89; panel F). No significant differences were found in early visual areas $\left(t=-1.13, p_{\mathrm{FDR}}=0.27\right.$, Cohen's $\mathrm{d}=0.35$; panel $\left.\mathbf{A}\right)$ and $\mathrm{PMC}\left(t=-1.91, p_{\mathrm{FDR}}=0.08, \mathrm{Cohen}\right.$ 's d $=$ 0.65 ; panel D). NS=Not significant; $* p_{\mathrm{FDR}}<0.05 ; * * p_{\mathrm{FDR}}<0.01$. 


\section{Similar connectivity pattern-mediated event integration is correlated with subsequent retrieval success}

Next, we investigated the association between connectivity patterns - a different multivariate method to characterise neural representations - and subsequent memory retrieval. Within-region multi-voxel connectivity patterns were calculated by a voxel-by-voxel pairwise correlation matrix resulting from the correlations between time courses of all voxels within a given region. This represents the relative correlation structure between all voxels in a certain region during event processing. We first calculated the event-specific within-region connectivity patterns for two sequential events, and then we quantified the similarity between connectivity patterns across event boundaries also using Pearson's r. Contrasting similarities of connectivity patterns of subsequently remembered and forgotten events allowed us to examine how transitions in connectivity patterns contribute to memory formation. We found higher connectivity pattern similarity for subsequently remembered compared to forgotten events in the early auditory area $\left(t=2.9, p_{\mathrm{FDR}}=0.02\right.$, Cohen's $\mathrm{d}=0.72$, Figure 4B $)$, visual areas $\left(t=3.34, p_{\mathrm{FDR}}=0.01\right.$, Cohen's d $=0.74$, Figure 4A), hippocampus $\left(t=3.39, p_{\mathrm{FDR}}=0.01\right.$, Cohen's $d=0.73$, Figure 4E), and PMC $\left(t=2.79, p_{\mathrm{FDR}}=0.02\right.$, Cohen's d $=0.47$, Figure 4D $)$. The same contrast was not significant for $\operatorname{mPFC}\left(t=1.22, p_{\mathrm{FDR}}=0.23\right.$, Cohen's $\mathrm{d}=0.25$, Figure 4C $)$ and $\mathrm{pPHG}\left(t=1.36, p_{\mathrm{FDR}}=0.22\right.$, Cohen's $\mathrm{d}=$ 0.30 , Figure 4F).

The same set of additional statistical tests was applied to the connectivity pattern analyses. (1) Event pairs were divided into four categories based on memory (i.e., $F F, F R, R F, R R$ ), and connectivity pattern similarities were compared between these four categories. Consistent with our main analyses, hippocampal connectivity pattern similarities are higher for RR pairs compared to FF pairs $(\mathrm{t}=3.85$, $\left.p_{\text {raw }}=0.002\right)$. This is also true for early auditory area $\left(\mathrm{t}=2.56, p_{\text {raw }}=0.02\right)$, early visual area $(\mathrm{t}=3.70$, $\left.p_{\text {raw }}=0.002\right)$ and PMC ( $\left.\mathrm{t}=2.11, p_{\text {raw }}=0.05\right)($ Figure S12). (2) Permutation tests examined the specificity of subsequent memory effects to actual event boundaries (as opposed to randomly generated pseudo boundaries) (Figure S13). (3) When memory labels were assigned randomly to connectivity patterns, the 
reported subsequent memory effects disappeared (Figure S14). (4) The event-specific correlational analysis demonstrated that the recall rate positively correlated with connectivity pattern similarity in the early auditory area $\left(\mathrm{r}=0.327, p_{\text {raw }}=0.022\right)$, visual areas $\left(\mathrm{r}=0.35, p_{\text {raw }}=0.014\right)$, hippocampus $(\mathrm{r}=0.301$, $\left.p_{\text {raw }}=0.036\right), \operatorname{PMC}\left(\mathrm{r}=0.341, p_{\text {raw }}=0.017\right)$, and pPHG $\left(\mathrm{r}=0.341, p_{\text {raw }}=0.017\right)$ (Figure S15). This supports our main findings, suggesting that events with higher connectivity pattern similarity with the subsequent event in these ROIs were more likely to be recalled. (5) Individual difference analyses revealed the same trends in the same direction as within-subject the within-subject contrast analyses: participants with higher connectivity pattern similarity in the early auditory $\left(\mathrm{r}=0.45, p_{\text {raw }}=0.06\right)$ and hippocampus ( $\left.\mathrm{r}=0.40, p_{\text {raw }}=0.10\right)$ were more likely to perform better at the memory test (Figure S11). (6) Mixed-effects models without $\left(\mathrm{F}=3.68, p_{\text {raw }}=0.05, \mathrm{R}^{2}=0.003\right)$ and with covariates $\left(\mathrm{F}=2.81, p_{\text {raw }}=0.09\right)$ showed the same tendency that higher hippocampal pattern connectivity was associated with better memory (Table S3). 
A

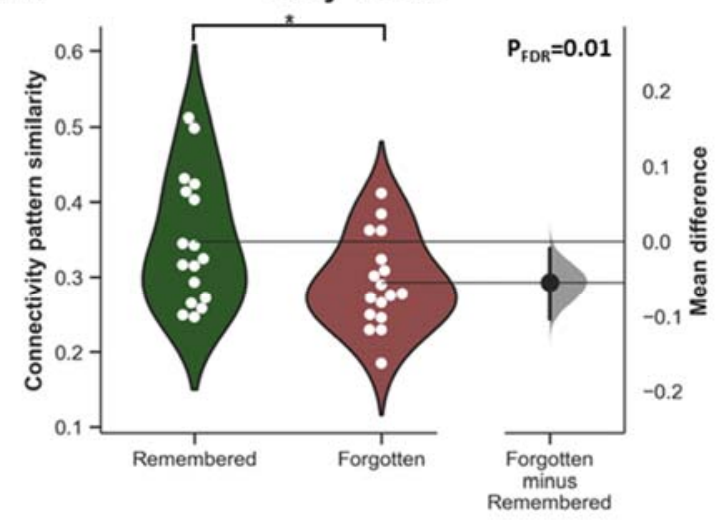

C

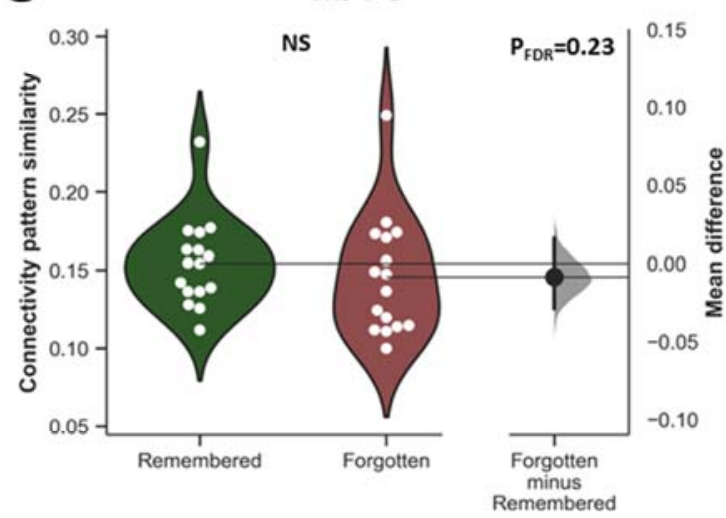

$\mathbf{E}$

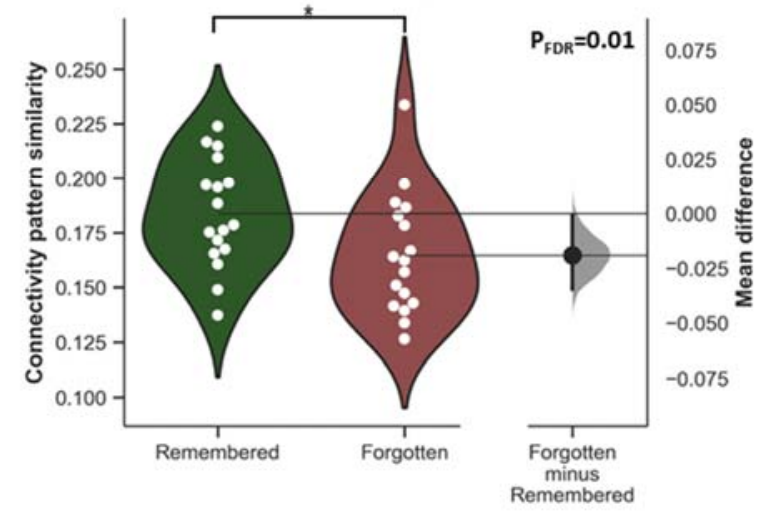

B

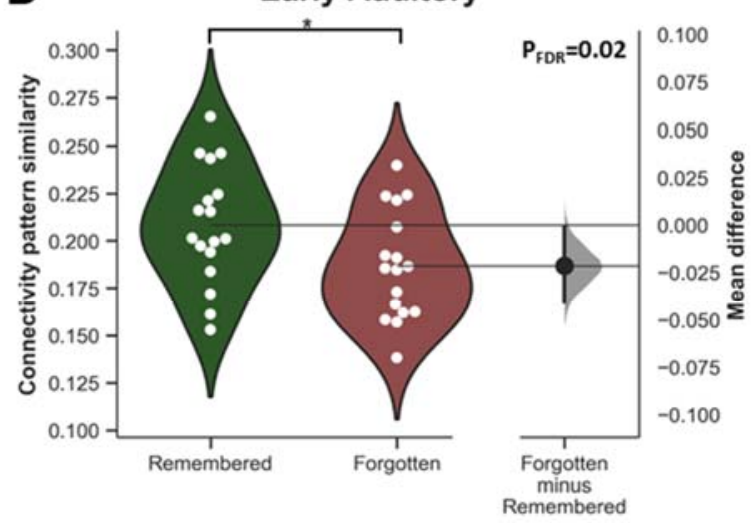

D

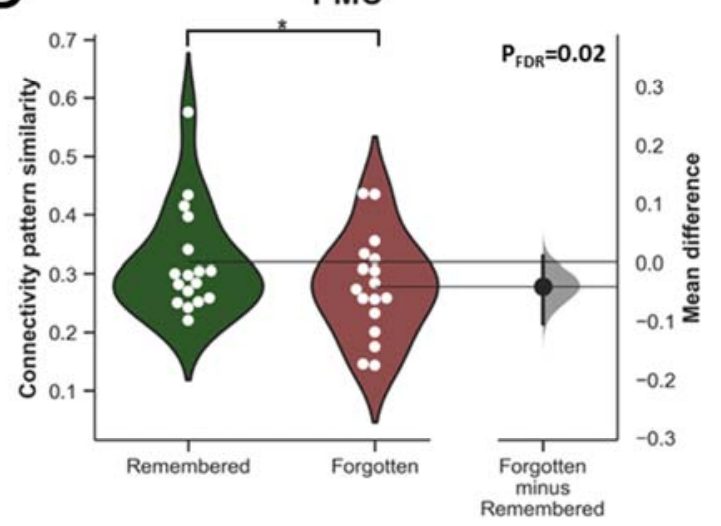

$\mathbf{F}$

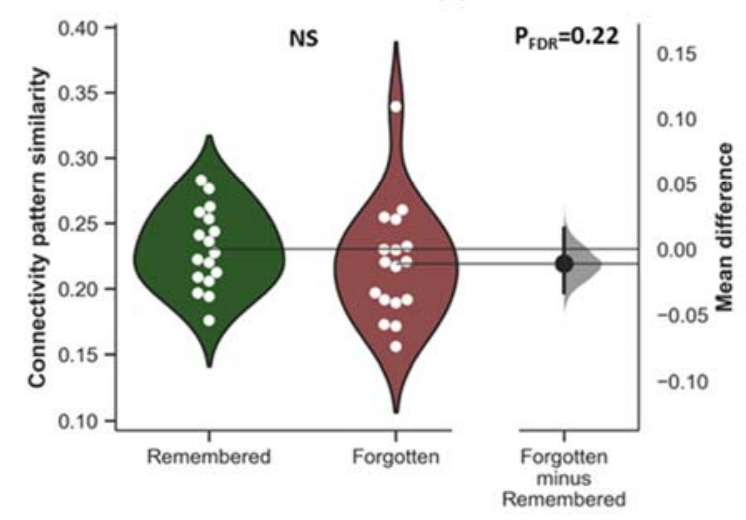

Figure 4. Association between connectivity pattern similarities of six ROIs and subsequent memory recall. We compared connectivity pattern similarities of sequential event pairs based on subsequent memory performance of the first event (Remembered vs. Forgotten) across six ROIs. For panel A-F, connectivity pattern similarities for Remembered events are displayed on the left (green), while similarities for Forgotten events are displayed on the right (red). For each comparison, a separate axis displays the mean difference. The curve (gray) indicates the resampled distribution of the mean difference generated via bootstrapping. The solid vertical line attached to the curve represents the mean difference as a $95 \%$ bootstrap confidence interval. We found significantly higher connectivity pattern similarity for Remembered (green) vs. Forgotten (red) event pairs in the early auditory area $\left(t=2.9, p_{\mathrm{FDR}}=0.02\right.$, Cohen's $\mathrm{d}=0.72$, panel $\left.\mathbf{B}\right)$, visual areas $\left(t=3.34, p_{\mathrm{FDR}}=0.01\right.$, Cohen's $\mathrm{d}=0.74$, panel A), hippocampus $\left(t=3.39, p_{\mathrm{FDR}}=0.01\right.$, Cohen's $\mathrm{d}=0.73$, panel $\left.\mathbf{E}\right)$, and PMC $\left(t=2.79, p_{\mathrm{FDR}}=0.02\right.$, Cohen's $\mathrm{d}=0.47$, panel D). No significant differences were found in $\operatorname{mPFC}\left(t=1.22, p_{\mathrm{FDR}}=0.23\right.$, Cohen's d $=0.25$, panel $\left.\mathbf{C}\right)$ and $\mathrm{pPHG}(t=1.36$, $p_{\mathrm{FDR}}=0.22$, Cohen's d $=0.30$, panel $\left.\mathbf{F}\right) . \mathrm{NS}=$ Not significant; $* p_{\mathrm{FDR}}<0.05$. 


\section{Similar connectivity pattern-mediated event integration preserves sequential memory of events in later retrieval}

So far we have shown the opposite association between our two multivariate neural pattern measures and subsequent memory performance: distinct activation patterns, but similar within-region connectivity patterns across events in the early auditory cortex and hippocampus predict retrieval success. This pattern of results suggests that the connectivity pattern may integrate events into a continuous sequence. To directly test this hypothesis, we examined the relationship between connectivity pattern similarity and sequential order of subsequent recall. We reasoned that if the connectivity patterns remain stable across event boundaries, events should tend to be recalled in the correct sequential order. We compared the mean connectivity pattern similarities for in-order and out-of-order events. Controlling for multiple comparisons, we found that connectivity pattern similarity in early visual cortex to be larger for in-order compared to out-of-order events $\left(t=3.16, p_{\mathrm{FDR}}=0.03\right.$, Cohen's d $=0.47$, Figure 5A $)$. Similar trends that did not survive correction for multiple comparisons were detected in the hippocampus $\left(t=-2.43, p_{\text {raw }}=\right.$ $0.026, p_{\mathrm{FDR}}=0.08$, Cohen's $\mathrm{d}=0.53$, Figure 5E $)$, auditory area $\left(t=-2.08, p_{\mathrm{raw}}=0.053, p_{\mathrm{FDR}}=0.084\right.$, Cohen's $d=0.46$, Figure 5B $)$ and posterior parahippocampal gyrus $\left(t=-2.05, p_{\text {raw }}=0.056, p_{\mathrm{FDR}}=0.084\right.$, Cohen's $d=0.36$, Figure 5F $)$. No such effect was observed in the $\mathrm{mPFC}\left(t=-1.35, p_{\mathrm{FDR}}=0.19\right.$, Cohen's $\mathrm{d}=0.19$, Figure 5C), and PMC $\left(t=-2.05, p_{\mathrm{FDR}}=0.12\right.$, Cohen's $\mathrm{d}=0.33$, Figure 5D $)$. It is worth mentioning that our method cannot completely disentangle the neural effects of "event memory" and "order memory". When we further fully control for the "event memory" by restricting analyses on two neighboring events that were both recalled, we did not found the different connectivity pattern similarity levels between "in-order" and "out-of-order" events among six ROIs (Supplemental Materials-alternative order memory analysis). 
A

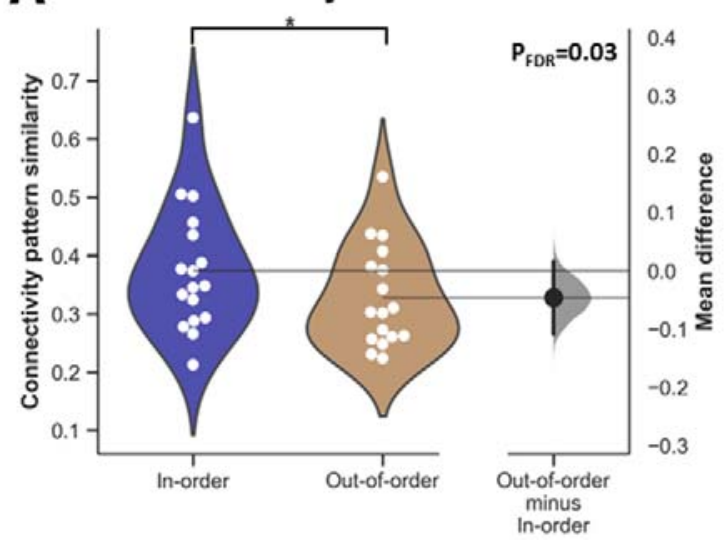

C

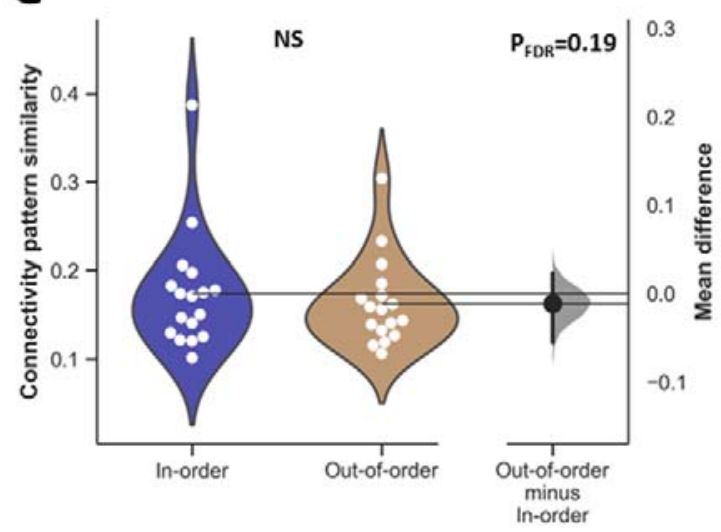

$\mathbf{E}$

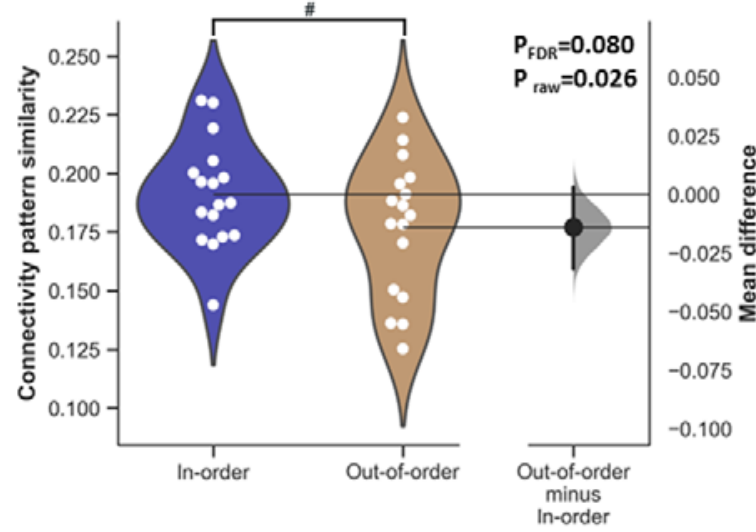

B

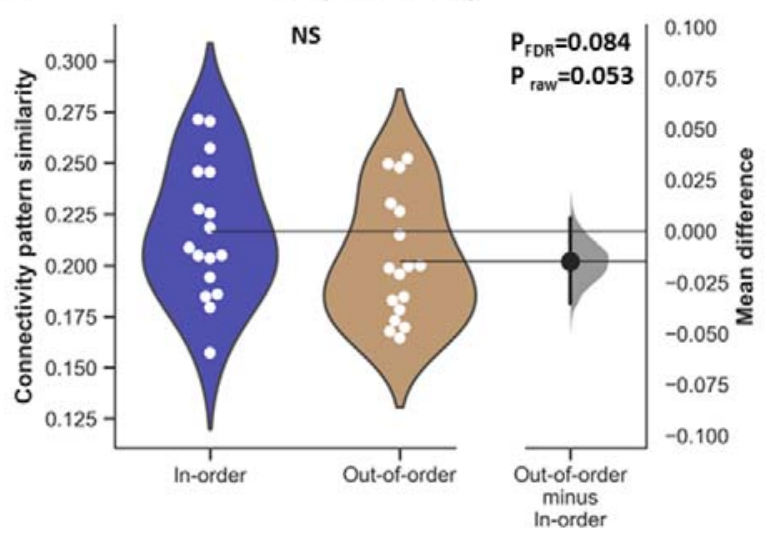

D

PMC

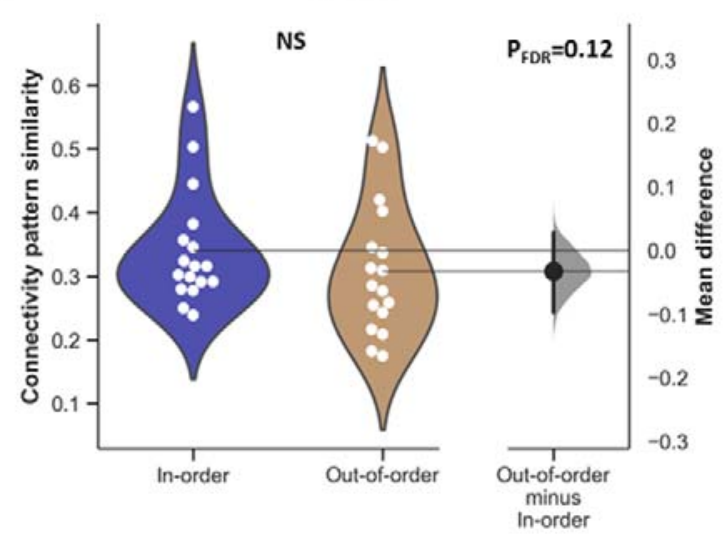

$\mathbf{F}$

Post. Parahipp.

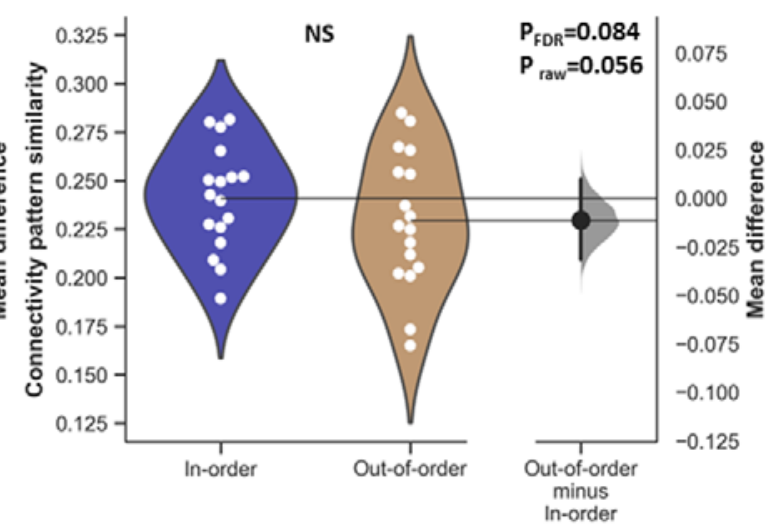

Figure 5. Association between connectivity pattern similarities of six ROIs and sequential order of memory recall. We compared connectivity pattern similarities of sequential event pairs (In-order vs. Out-of-order) based on sequential memory performance of the first event across six ROIs. For panel A-F, connectivity pattern similarities for In-order events are displayed on the left $(B L U E)$, while similarities for Out-of-order events are displayed on the right (BROWN). Early visual areas $(t=3.16$, $p_{\text {FDR }}=0.03$, Cohen's d $=0.47$, panel A) demonstrated higher connectivity pattern similarity for the In-order events compared to Out-of-order events. A similar trend was also detected in the hippocampus $\left(t=-2.43, p_{\text {raw }}=0.026\right.$, Cohen's $\mathrm{d}=0.53$, panel $\left.\mathbf{E}\right)$, but it did not survive FDR correction $\left(p_{\mathrm{FDR}}=0.08\right)$. We also found modest, non-significant trends in the early auditory area $(t=-$ $2.08, p_{\text {raw }}=0.053, p_{\mathrm{FDR}}=0.084$, Cohen's $\mathrm{d}=0.46$, panel $\left.\mathbf{B}\right)$ and posterior parahippocampal gyrus $\left(t=-2.05, p_{\text {raw }}=0.056, p_{\mathrm{FDR}}=\right.$ 0.084 , Cohen's $d=0.36$, panel F). No similar effects were detected in $\operatorname{mPFC}\left(t=-1.35, p_{\text {FDR }}=0.19\right.$, Cohen's $d=0.19$, panel C $)$, and PMC $\left(t=-2.05, p_{\mathrm{FDR}}=0.12\right.$, Cohen's $\mathrm{d}=0.33$, panel D $)$. NS=Not significant; * $p_{\mathrm{FDR}}<0.05 ; \# p_{\mathrm{raw}}<0.05$. 


\section{Hippocampal activation and connectivity patterns change differently with event distance}

Among our six ROIs, we found converging evidence for a dissociation of event segmentation and integration in the hippocampus: lower activation pattern similarity, but higher connectivity pattern similarity was beneficial for memory formation. Building on these findings, we hypothesized that hippocampal activation patterns of neighboring events should be less similar than events that occur far apart. By contrast, hippocampal connectivity patterns of close events should be more similar than events with a long interval in between. Thus, we calculated the activation and connectivity pattern similarity between all possible combinations of event pairs ('Event A' and 'Event B') within all 50 events (Figure 6A and 6D). For all pairs of events with the same event distance (e.g., separated by four events), we calculated the mean similarity measure for activation pattern and connectivity pattern separately. This calculation was repeated for all possible event distances. To ensure reliable estimations of pattern similarities, we only present the similarities of distances with at least ten event pairs $(\mathrm{d} \leq 40)$ in the main text. (Complete calculations can be found in Figure S16)

We analysed the hippocampal activation and connectivity patterns separately. First, our activation pattern analysis found that the shorter the event distance, the more distinct the hippocampal activation patterns ( $\mathrm{r}$ $=0.21, p_{\text {raw }}=1.8 \times 10^{-8} ;$ Figure $6 \mathbf{B}$ and $\left.\mathbf{S 1 6 A}\right)$. This positive correlation was largely driven by the negative similarity values between events that occurred close to each other (i.e., events with a distance smaller than four). Furthermore, we found that subsequent memory recall of Event A modulated the relationship between event distance $(\mathrm{d}=1-4)$ and activation pattern similarity (ANOVA with event $\mathrm{A} \times$ distance interaction: $F(3,48)=10.1, p<0.001$; Figure 6C). That is, hippocampal activation pattern similarities increased as the event distance changes from 1 to 4 , but only if event A was later recalled $\left(F_{\text {remembered }}(3,48)=9.54, p<0.001 ; F_{\text {forgotten }}(3,48)=1.35, p=0.268\right)$.

Second, our connectivity pattern analysis found that the shorter the event distance, the more similar the hippocampal connectivity patterns $\left(\mathrm{r}=-0.439, p_{\mathrm{raw}}=1.8 \times 10^{-33}\right.$; Figure $6 \mathbf{E}$ and S16B). Furthermore, we found a significant interaction between event A recall and distance $(F(19,304)=2.37, p=0.001)$, and a 
significant main effect of event $\mathrm{A}(F(1,16)=7.53, p=0.014)$. That is, if event $\mathrm{A}$ was recalled later, its hippocampal connectivity pattern was more similar to any other event in the sequence, compared to when event A was not successfully recalled (Figure 6F). This suggests that if connectivity patterns between pairs of events are more similar, for both short and long distances, then events are more likely to be successfully encoded.

Several time-dependent artifacts may contribute to signals in hippocampal event distance analyses (e.g., temporal distance, temporal filtering). These are unlikely to explain the subsequent memory effects we observed, but we ran several further analyses to limit their influence. First, (1) evaluate the effects of these potential artifacts (i.e., temporal distance, and temporal filtering) on the event distance analysis (Figure S18-S19). We investigated how hippocampal pattern similar change with event distance when the temporal distance between events (i.e., the number of TRs) was controlled and when different cutoffs (i.e., $140 \mathrm{~s}, 280 \mathrm{~s}, 420 \mathrm{~s}, 560 \mathrm{~s}, 600 \mathrm{~s})$ for high-pass filtering were applied to the time-series; Second, we performed a permutation test to validate the subsequent memory effects in the event distance analysis. We shuffled memory labels (i.e., $R$ and $F$ ) randomly and performed the event distant analysis for each permutation. Third, event distance analysis was also applied to ROIs beyond the hippocampus to probe whether presented effects are hippocampal-specific. Results can be found in Figure S20-S21. These control analyses together demonstrated that (1) main findings were robust to artifacts, (2) relationship between event distance and neural similarity was indeed modulated by memory, (3) similar relationship between event distance and neural similarity were presented in other ROIs as well but it interacted with subsequent memory in a region-specific manner. 


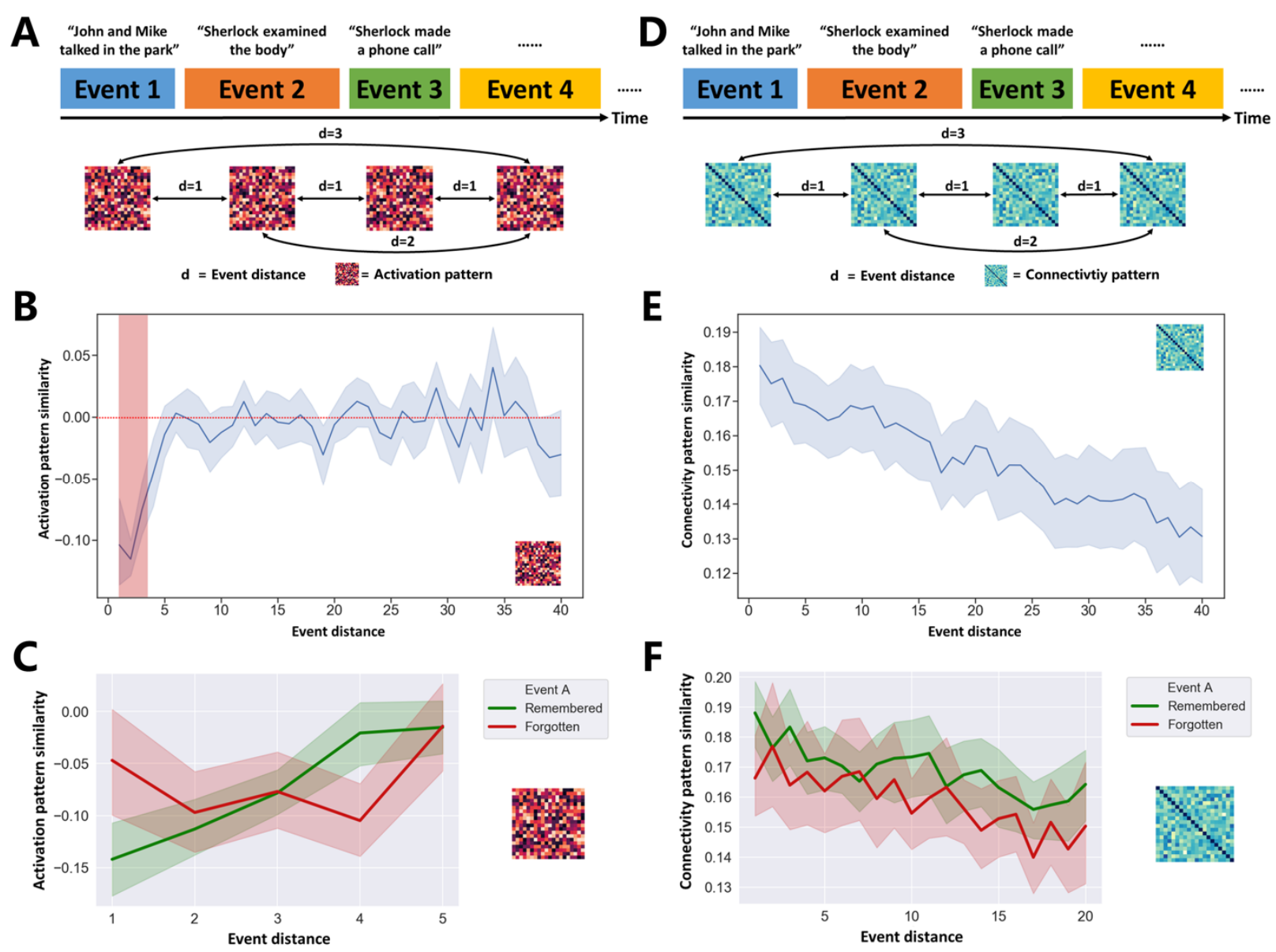

Figure 6. Hippocampal pattern similarity changes with event distance. (A) Hippocampal activation patterns were generated for all 50 events. We calculated activation pattern similarities between sequential events (event distance $=1$ ) and all possible combinations of non-sequential event pairs (event distance > 1). (B) Hippocampal activation patterns between pairs of events were significantly dissimilar for events separated by a distance of less than 4 (red shadow). (C) Memory performance modulated the distance-activation pattern similarity relationship. If the first event (Event A) of the pair was successfully encoded, activation pattern similarities of the event pair increased with event distance (green line). (D) Hippocampal connectivity patterns were generated for all possible combinations of event pairs. (E) Event pairs with shorter event distance had more similar hippocampus connectivity patterns. At the same time, similarities of hippocampus connectivity patterns are higher than 0 regardless of event distance. (F) Memory performance modulated distance-connectivity pattern similarity relationship. If the first event (Event $A)$ of the event pair was successfully encoded, connectivity pattern similarities of the event pair are enhanced regardless of their event distance. For panel B-F, error bands (i.e., light shadow around the solid line) represent the $95 \%$ confidence interval of the mean.

\section{Subregions of the prefrontal cortex perform event segmentation and integration}

Our ROI-level analyses found that (1) distinct hippocampal activation patterns were associated with better event memory; (2) similar hippocampal connectivity patterns were beneficial for event memory; (3) although not surviving multiple comparison correction, similar hippocampal connectivity patterns tended to preserve the sequential order of events (Figure 7A). To investigate whether these relationships are 
present in other brain regions beyond our six ROIs, we ran a region-based searchlight version of our pattern similarity analysis to identify overlapping event segmentation and integration computations across neorcortical regions. In sum, we investigated three potential relationships between neural pattern similarity and subsequent retrieval separately. First, we identified brain regions whose lower activation pattern similarities across events were associated with retrieval success (Figure S22A). Next, we mapped the association between higher connectivity pattern similarities and retrieval success in each region (Figure S22B). Then, we identified the regions, which demonstrated a positive association between connectivity pattern similarities and order memory (Figure S22C).

To identify brain regions that may support all three neural computations (like the hippocampus), we overlapped spatial patterns for these three effects (all $p_{\mathrm{FDR}}<0.05$ ). This revealed a set of brain regions including relatively large clusters (at least 50 voxels) in the $\mathrm{mPFC}$, right inferior frontal gyrus (IFG), anterior/middle cingulate cortex and supplementary motor area (SMA), left inferior temporal gyrus (ITG) and left insular (Figure 7B). These results suggest that this network of cortical regions may use the same neural processes to perform event segmentation and integration as the hippocampus during continuous memory encoding. 
bioRxiv preprint doi: https://doi.org/10.1101/2020.03.14.990002; this version posted December 12, 2020. The copyright holder for this preprint (which was not certified by peer review) is the author/funder, who has granted bioRxiv a license to display the preprint in perpetuity. It is made available under aCC-BY-ND 4.0 International license.

\title{
A
}

\author{
Activation Pattern Similarity $\downarrow$
}

\& Event Memory $\uparrow$

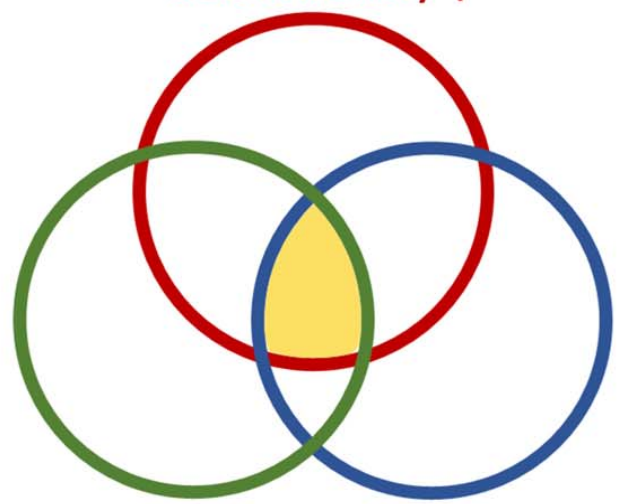

Connectivity Pattern Similarity $\uparrow$

\& Event Memory $\uparrow$

Connectivity Pattern Similarity $\uparrow$

\& Order Memory $\uparrow$

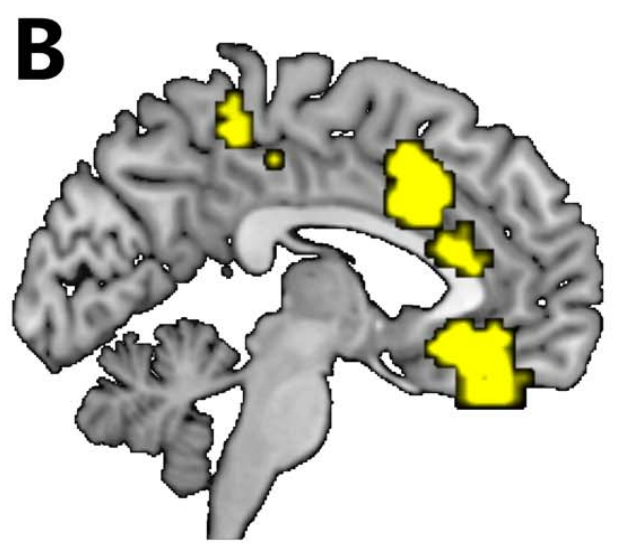

$x=-5$

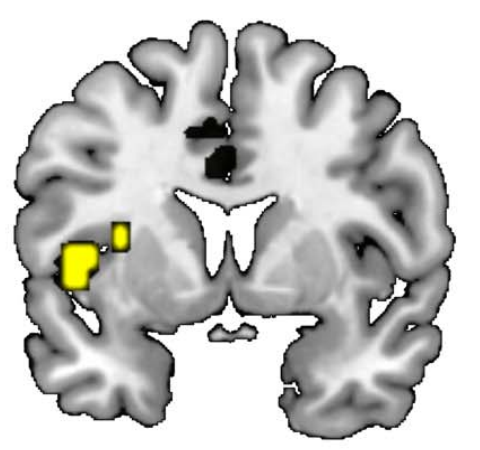

$y=6$

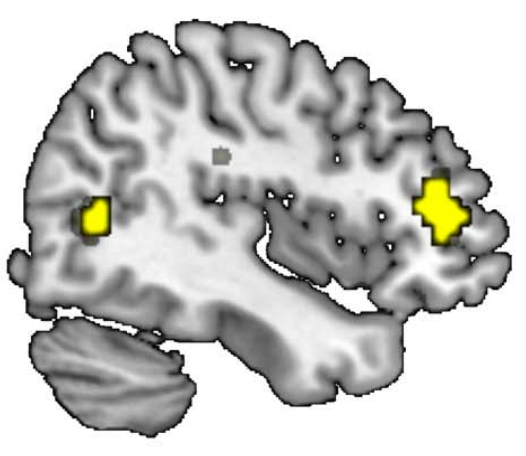

$x=-42$

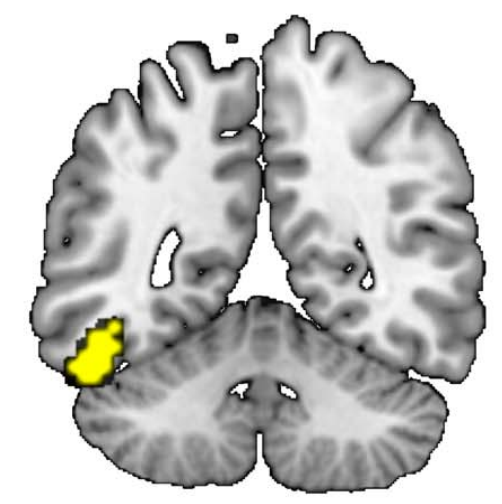

$y=51$

Figure 7. Identifying overlapping event segmentation and integration computations across the neocortex. (A) We identified three relationships between neural pattern similarity and subsequent memory in the hippocampus. (B) Similar to the hippocampus, overlapping event segmentation and integration computations were found in a network of brain regions including the medial prefrontal cortex (mPFC), right inferior frontal gyrus (IFG), anterior/middle cingulate cortex and supplementary motor area (SMA), left inferior temporal gyrus (ITG), and left insular $\left(p_{\text {FDR }}<0.05\right.$ across 1000 parcels, cluster size $\left.>=50\right)$. 


\section{Hippocampal neural pattern similarities were correlated with memory formation in a replication}

\section{dataset}

We analyzed an independent movie watching-recall dataset (Kurby and Zacks 2018) (i.e., replication dataset) to conceptually replicate reported associations between neural similarity and memory formation in the discovery dataset. Discovery dataset and replication differed in several aspects in terms of data acquisition protocol (See Methods), and therefore, provides the opportunity to test the generalization. Participants watched five short movies inside the MRI scanner and returned to the lab several days (mean=3.4 days) later for behavioral testing. During the behavioral session, participants also performed the segmentation task to generate participant-specific event boundaries for each movie clip (Figure 8A). These participant-specific boundaries were used as onsets/offsets of events to calculate event-specific fMRI activation patterns and connectivity patterns, and then neural similarities across boundaries within each movie clip (i.e., "within-movie" method). The estimation of neural similarities using the "withinmovie" method is identical to the counterpart used in the discovery dataset. Furthermore, neural similarities were also calculated across different movie clips (i.e., "between-movie" method) (Figure 8B). We correlated clip-specific neural similarity measures with all three different memory measures (i.e., recognition, recall, and order memory). Results from the replication dataset that survived FDR-correction are summarized in Figure 8C alongside the discovery dataset results in Figure 8D (for full results see Figure S23-S24 and Table S6-S7).

Using the "within-movie" method, we found that lower activation pattern similarity in the hippocampus during movie watching correlated with better recognition memory $\left(\mathrm{r}=-0.14, p_{\text {raw }}=0.028, p_{\mathrm{FDR}}=0.033\right)$. Similar correlations were found in early auditory cortex $\left(\mathrm{r}=-0.17, p_{\text {raw }}=0.007, p_{\mathrm{FDR}}=0.021\right), \mathrm{mPFC}(\mathrm{r}=-0.14$, $\left.p_{\text {raw }}=0.025, p_{\mathrm{FDR}}=0.033\right), \mathrm{PMC}\left(\mathrm{r}=-0.17, p_{\text {raw }}=0.006, p_{\mathrm{FDR}}=0.021\right)$, and $\mathrm{pPHG}\left(\mathrm{r}=-0.16, p_{\text {raw }}=0.01\right.$, $\left.p_{\mathrm{FDR}}=0.022\right)$. Thus, the relationship between subsequent retrieval success and lower activation pattern similarity in the hippocampus as well as early auditory cortex, $\mathrm{mPFC}$, and pPHG was replicated across our two datasets (Figure 8C-D). 
Activation pattern similarity in the mPFC and PMC also correlated with additional memory measures (mPFC: with order memory $\left(\mathrm{r}=-0.19, p_{\text {raw }}=0.002, p_{\text {FDR }}=0.012\right)$; PMC: with order memory $(\mathrm{r}=-0.16$, $\left.p_{\text {raw }}=0.009, p_{\mathrm{FDR}}=0.027\right)$ and recall $\left.\left(\mathrm{r}=-0.17, p_{\mathrm{raw}}=0.006, p_{\mathrm{FDR}}=0.036\right)\right)$. However, connectivity pattern similarities estimated by the "within-movie" method did not associate with any memory measures. This may because durations were too short based on participants' segmentation: event durations were around 60s in the discovery dataset, compared with 6-10s in the replication dataset. We propose that 3-5 TRs are insufficient to be viewed as "encoding context" and enable meaningful connectivity analyses. To explore further, we next regarded each movie clip (2 to $6 \mathrm{~min}$ ) as an event and performed the calculation of connectivity patterns using the "between-movie" method. We found that higher connectivity pattern similarity across movie clips in the hippocampus $\left(\mathrm{r}=0.17, p_{\mathrm{raw}}=0.013, p_{\mathrm{FDR}}=0.019\right)$, early auditory cortex $\left(\mathrm{r}=0.2, p_{\text {raw }}=0.003, p_{\mathrm{FDR}}=0.006\right), \mathrm{mPFC}\left(\mathrm{r}=0.15, p_{\text {raw }}=0.036, p_{\mathrm{FDR}}=0.043\right), \mathrm{PMC}\left(\mathrm{r}=0.26, p_{\text {raw }}<0.001\right.$, $\left.p_{\mathrm{FDR}}<0.006\right)$, and $\mathrm{pPHG}\left(\mathrm{r}=0.21, p_{\mathrm{raw}}=0.002, p_{\mathrm{FDR}}=0.006\right)$ was associated with better recall performance. Therefore, the "between-movie" method showed that the relationship between retrieval success and higher connectivity pattern similarity in the hippocampus, PMC, and early auditory cortex was also replicated across two datasets (Figure 8C-8D).

Furthermore, we found modest evidence (i.e., $p_{\text {raw }}$ value around 0.05 ) to support that higher connectivity pattern similarity in these ROIs tended to associated with better order memory performance (early auditory cortex $\left(\mathrm{r}=-0.137, p_{\text {raw }}=0.048\right), \mathrm{mPFC}\left(\mathrm{r}=-0.12, p_{\text {raw }}=0.08\right), \mathrm{PMC}\left(\mathrm{r}=-0.135, p_{\text {raw }}=0.052\right)$ and $\mathrm{pPHG}$ $\left.\left(\mathrm{r}=-0.12, p_{\text {raw }}=0.08\right)\right)$. Across two datasets, connectivity pattern similarities of the early auditory cortex and pPHG were associated with better order memory.

In sum, the relationships between hippocampal activation and connectivity pattern similarity and episodic memory formation were replicated across two datasets. However, higher hippocampal connectivity pattern similarity was associated with better order memory only in the discovery dataset, but not in the replication dataset. Also, our "within-movie" connectivity analysis did not reveal the subsequent memory effect, but that could be due to experimental design (ie., short events). When we analyzed connectivity patterns on a longer timescale with "between-movie" analysis, then memory effects got replicated. 
A Session1: Movie Watching \& fMRI Scanning

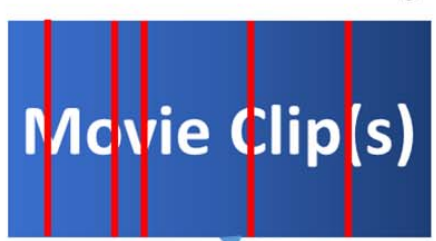

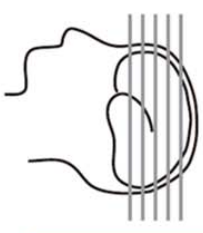

Participant-specific event boundary

\section{Several Days \\ Later \\ (mean= \\ $3.4 \mathrm{ds}$ )}

\section{Session2: Behavioral testing}

Memory

Tests

Event

Segmentation

B

Within Movie Clip(s)

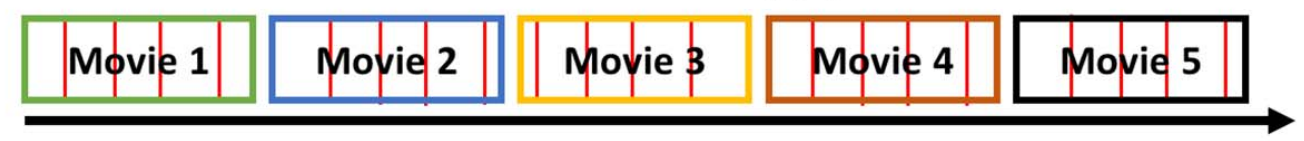

Between Movie Clip(s)
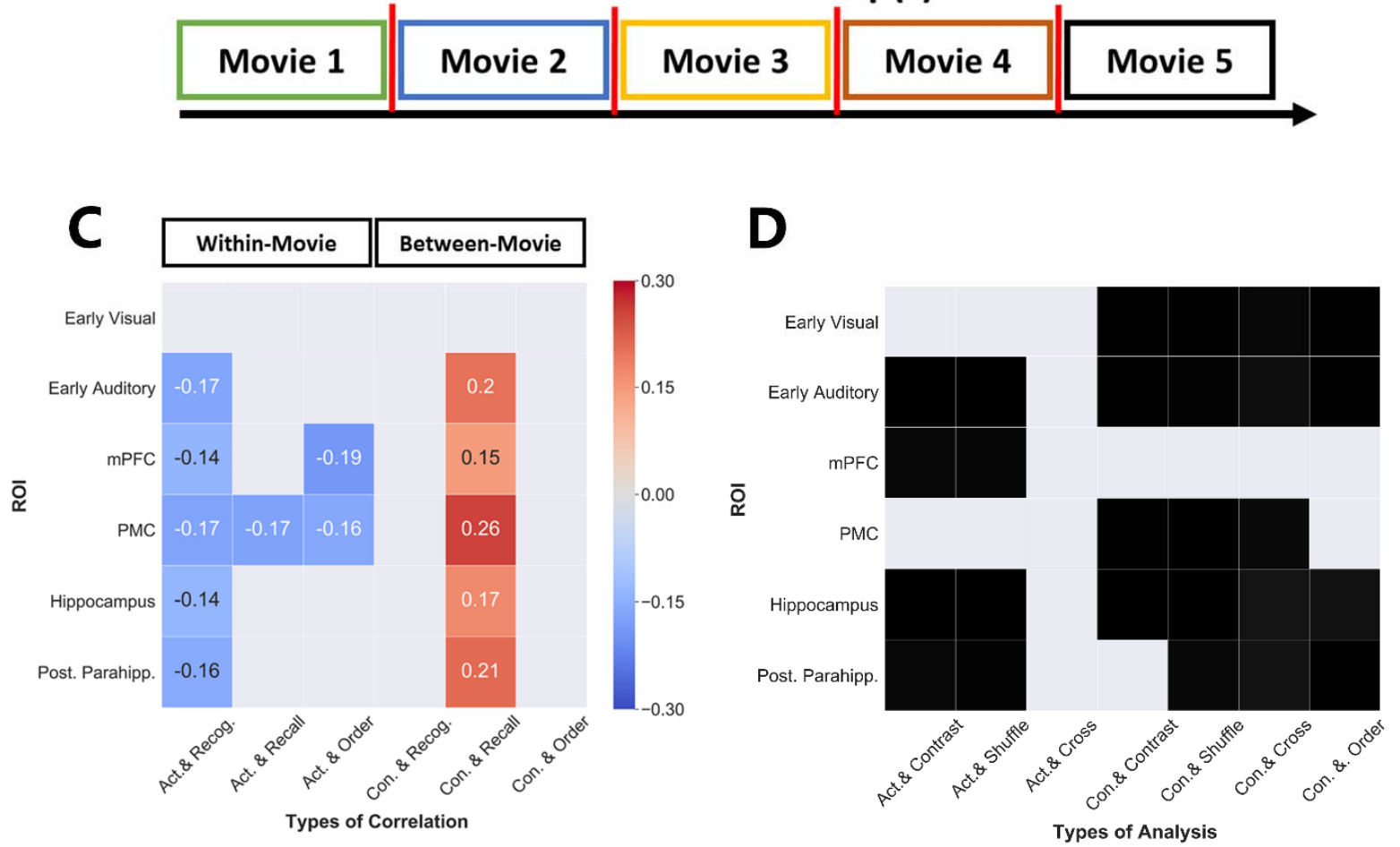

Figure 8 Experimental design as well as data analyses in the replication dataset, and comparison between results from two datasets. (A) In the replication dataset, participants watched movie clips inside the MRI scanner (session1) and returned to the lab several days (mean=3.4 days) later for behavioral testing (session2) including both segmentation and memory tests. (B) Two methods (i.e., within-movie and between-movie) were used to estimate neural pattern similarity. For the within-movie method, participant-specific boundaries were used to define events within movies while for the between-movie method, each movie clip was regarded as an individual event. (C) Correlations between ROI-specific neural pattern similarity and memory measures in the replication dataset. Correlation coefficients are presented for significant tests that survived FDR-correction. Full results from the replication dataset can be found in Figure S22-S23 and Table S5-S6. (D) Significant tests in the discovery dataset, with solid blocks for those that survived FDR-correction. Act.=Activation pattern; Con.=Connectivtiy pattern; Recog.=Recognition; Contrast=contrast between Remembered and Forgotten; Shuffle=test with shuffled event boundaries; Cross=cross-event analyses.

\section{Discussion}


To successfully form memories of our life experiences, we need to segregate continuous experience into events (Baldassano et al. 2017; Zacks 2020) and integrate those events across their boundaries into a coherent narrative (Griffiths and Fuentemilla 2020). Here we show that distinct hippocampal activation patterns, but similar hippocampal connectivity patterns across event boundaries, facilitate these two vital episodic memory functions. We propose that distinct activation patterns reflect event segmentation while similar connectivity patterns integrate separately represented events into a narrative. Supporting this role of connectivity patterns for event integration, we found that similar hippocampal connectivity patterns were relevant for the correct sequential order of subsequent retrieval. Our whole-brain analysis demonstrates that similar neurocomputations were performed by a network of cortical regions, in particular for the mPFC. Finally, these results were further validated using an independent moviewatching recall dataset, in which different stimuli and memory measures were utilized. Among the same set of ROIs, hippocampal activation and connectivity pattern similarity showed consistent relationships with memory across these two datasets. Overall, these results suggest that both hippocampal and medial prefrontal event segmentation and integration support memory formation of continuous experience.

Using multivoxel pattern analysis, we found that distinct local activation patterns across event boundaries in the early auditory area, mPFC, posterior parahippocampal gyrus, and hippocampus, were associated with better subsequent memory, indexed by more distinct activation patterns between two adjacent events. The ability to segment continuous experience at the behavioral level has been linked to successful memory encoding (Sargent et al. 2013) and compelling evidence suggested that the hippocampus is activated around event boundaries (Ben-Yakov and Dudai 2011; Ben-Yakov et al. 2013; DuBrow and Davachi 2013; Ben-Yakov and Henson 2018). This hippocampal activity has been proposed to be associated with a hippocampal segmentation process, but how the hippocampus represents two separate events, and whether the corresponding neural representations are relevant for memory remained unclear. Our findings suggest that the hippocampus and other brain regions (e.g., mPFC) segment events by representing them with two distinct patterns of activity. This is similar to the role of the hippocampus in pattern separation: when 
similar experiences need to be discriminated and encoded, the underlying hippocampal neural representations tend to be dissimilar (Bakker et al. 2008; Yassa and Stark 2011). This neural phenomenon has typically been studied to show how the brain separates perceptually similar stimuli (i.e., images). Here, our findings indicate that a similar pattern separation occurs for events during the continuous experience and this determines subsequent memory for those events. That is to say, the episodic memory system may use 'orthogonalized' neural representations to encode two events for the purpose of event segmentation. Further, we show these 'orthogonalized' neural representations are potentially event-distance dependent: the hippocampus only generates consecutive dissimilar patterns when events occur relatively close in time. Taken together, this suggests the existence of a brain network (mainly hippocampus and mPFC) for the continuous segmentation of ongoing experience, and the degree of separate neural event representation for nearby events is relevant for memory formation.

Complementing this, we found that more similar within-region connectivity patterns of several regions across event boundaries, including again the early auditory area and hippocampus, were associated with the better subsequent recall. Compared to local activation patterns (Cohen et al. 2017; Xue 2018), withinregion connectivity patterns are a less used multivariate approach. Recently, Tambini and Davachi proposed that both activation and connectivity patterns could be used to capture neural states during memory encoding and reactivation, but connectivity patterns tend to encode contexts or states instead of particular perceptual inputs (Tambini and Davachi 2019). Our results support this notion: activation patterns were event-specific (Chen et al. 2017) and dissimilar for neighboring events, while connectivity patterns were relatively stable across event boundaries. Importantly, the stability of connectivity patterns positively associated with memory formation. Therefore, the function of similar connectivity patterns could be to integrate segmented and separately represented events into a coherent narrative. To provide further support for this idea, we examined the relationship between connectivity pattern similarity and sequential order during memory recall and found that higher cross-event connectivity pattern similarity (mainly in the hippocampus and pPHG) was associated with better retrieval of the sequential order of 
individual events. Results from the replication dataset, in which a more accurate order memory measure was adopted, still demonstrated the role of pPHG's connectivity pattern in promoting order memory. In general, these results revealed that the multi-voxel connectivity pattern can be used to predict how temporal sequences are represented in the human medial temporal lobe memory system. Interestingly, our activation and connectivity pattern measures were both modulated by event boundaries in a similar way (ie., greater within-event similarity compared with between-event similarity). But the way they interact with memory was different/opposite, with dissimilar patterns being better for activation, and similar patterns being better for connectivity.

Remembering the sequence of events is not only one of the critical features of episodic memory (Davachi and DuBrow 2015) but also highly relevant for other forms of sequence learning, for example, spatial memory and encoding of temporal information (Eichenbaum 2014; Bellmund et al. 2020). Animal studies have revealed the existence of hippocampal neurons (i.e., time cells (Manns et al. 2007), event-specific rate remapping cells (Sun et al. 2020)) that are causally involved in representing temporally structured experience. Single-cell recordings in the hippocampus of patients with pharmacologically intractable epilepsy showed that as a result of repeated viewing of the same video clips, neuronal activity in successive time segments became gradually correlated, and this potential measure of temporal binding predicted subsequent recall (Paz et al. 2010). This study revealed how the temporal relationship between current hippocampal activity and hippocampal activity that follows in time can be used to link successive events in humans. Other recent human fMRI studies revealed the role of the human hippocampalentorhinal region in representing the temporal sequence of experience across different paradigms and stimuli (Lositsky et al. 2016; Bellmund et al. 2019; Montchal et al. 2019; Thavabalasingam et al. 2019). Adding to this, our study tested the role of a new multivariate measure, connectivity pattern similarity, which could reflect the internal stability of neural states, in temporal sequence coding. We provided preliminary evidence that connectivity pattern similarity across event boundaries in the medial temporal lobe is involved in sequential memory. Future studies are needed to further investigate the precise 
mnemonic functions of different neural measures (e.g., activity pattern, within-region connectivity pattern, and system-level interaction between regions) during memory formation, in particular for encoding temporal structure during the continuous experience (Tambini and Davachi 2019).

Our ROI analysis highlights the two functions of the hippocampus in the separate representation of segmented events and the binding function that linked events into a narrative, and region-based searchlight analysis identified the role of subregions of the prefrontal cortex (e.g., mPFC, IFG), insular, and inferior temporal gyrus in event segmentation and integration during memory formation. The role of the mPFC in event integration is particularly thought-provoking. The mPFC is generally implicated in encoding and retrieval of episodic memories (Kim 2010; Rugg and Vilberg 2013). Among its variety of functions in learning and memory (Fernández 2017), the online integration of events we observed here is consistent with its function in the facilitation of associative inference (Zeithamova et al. 2012; Preston and Eichenbaum 2013; Schlichting et al. 2014; Schlichting and Preston 2015; Spalding et al. 2018), accumulation of knowledge (Kumaran et al. 2009; Berkers et al. 2018), and integration of new and prior knowledge (van Kesteren et al. 2010, 2013, 2014). We propose that the general mnemonic function of $\mathrm{mPFC}$ is to establish links between separate elements across time and space. Taken together, we found that the hippocampus-mPFC circuit performs event segmentation and integration during memory formation of continuous experience. These findings demonstrate the contribution of two complementary event processing mechanisms and underlying neural representations in episodic memory formation. The hierarchical network model of event segmentation proposes that higher-order regions receive event representations from lower-order perceptual regions, and then transfer these representations to the hippocampus for storage (Hasson et al. 2015; Baldassano et al. 2017). Our study suggested that event integration is another key cognitive process involved in event memory by showing how distinct event representations are integrated by similar connectivity patterns of hippocampus and mPFC.

Beyond the hippocampus, the role of sensory regions in event segmentation was also predicted by the hierarchical theory of event processing and supported by initial empirical evidence (Baldassano et al. 2017) 
and our analyses. As predicted by the theoretical model of event segmentation (Hasson et al. 2015), event segmentation signals could exist in multiple brain regions (including different levels of sensory regions), but interestingly, the continuous experience is represented at different time scales along with the hierarchical structure. The hippocampus may represent the event structure that aligns best to human-labels while lower-level sensory regions not only respond to this coarse segmentation but also create fine-scale segmentations within the individual event. This model was supported by human fMRI data (Baldassano et al. 2017): although sensory regions demonstrated more frequent transitions of activation patterns (i.e., more event boundaries) than higher-level regions, there are always some transitions matched with boundaries based on human labels(i.e., close to hippocampal segmentation). Therefore, it is not surprising that, when we choose human-labeled boundaries to analyses signals from sensory regions, we can still link their event segmentation measures to subsequent memory.

Our study, together with previous studies also combining human fMRI with naturalistic stimuli (Hasson et al. 2008; Baldassano et al. 2017; Chen et al. 2017), demonstrates the potential of this approach to advance our understanding of the human memory system, in particular for the formation of real-life memories. Similar paradigms and analyses can be easily adapted in clinical (e.g., memory and affective disorders) and developmental neuroimaging studies (e.g., children and older adults) to reveal changes related to disease or (mal)development. For example, fMRI-based event segmentation and integration measures could be used to probe how these processes are impaired in Alzheimer's disease and mild cognitive impairment, how they develop from childhood to adulthood and diminish in normal aging. In addition, connectivity patterns have the potential to inform our understanding of other cognitive operations that require integration of information, such as inferential reasoning (Preston and Eichenbaum 2013). However, due to the low temporal resolution of fMRI, the directionality of information flow between the neocortical regions of the 'hierarchical memory system' (Hasson et al. 2015) and the hippocampus remains unclear. Future application of deep-source magnetoencephalography (MEG) (e.g., Backus, Schoffelen, Szebényi, 
Hanslmayr, \& Doeller, 2016) or intracranial electroencephalography (iEEG) (e.g., Jafarpour, Griffin, Lin, \& Knight, 2019) with naturalistic memory paradigms may bridge this gap.

Despite the mentioned advantages of combining neuroimaging, naturalistic stimuli, and subsequent memory design to study episodic memory formation, there are also disadvantages worth noting, especially concerning the discovery dataset. For example, all participants viewed the same movie in the same order, therefore, memory for individual event and their sequential relationship cannot be isolated. Furthermore, in the discovery dataset, memory performance was relatively high, which means subsequent memory contrasts in some participants may not be well-powered. The inclusion of the replication dataset could potentially mitigate these concerns, revealing rather consistent neural effects in the hippocampus and parahippocampus across two datasets. Another disadvantage of naturalistic stimuli is that situational variables could influence how memorable each event is (ie., duration, music, location......). Some of them were indeed positively correlated with memory (e.g., event duration and arousal). We accounted for this in our mixed model analysis that included these situational factors, but the results were consistent with our main analyses. Therefore, our proposed mnemonic functions of the hippocampus are not likely to be the result of external factors.

In sum, we show that the hippocampus and $\mathrm{mPFC}$ may perform a dual function during naturalistic memory formation. Both regions segment events by representing them with distinct activation patterns, while also integrating those events by retaining similar connectivity patterns across events, enabling the representation of a coherent narrative. The ability to measure segmentation- and integration-related neural operations using fMRI opens new opportunities to investigate the mechanisms of memory encoding for real-life experience.

\section{Acknowledgement}


We thank Dr Janice Chen for sharing the dataset and ROI masks and suggestions on the searchlight analysis of this manuscript; Kirsten Rittershofer for proofreading this manuscript; Members of the Hasson and Norman laboratories for their efforts during data acquisition and data sharing. This work was supported by the Chinese Scholarship Council (PhD fellowship (201606990020)) to W.L), Ministerie van OCW (Sino-Dutch Bilateral Exchange Scholarship to YJS), and Nuffic Neso China (Orange Tulip Scholarship to YJS).

\section{References}

Backus AR, Schoffelen J-M, Szebényi S, Hanslmayr S, Doeller CF. 2016. Hippocampal-prefrontal theta oscillations support memory integration. Curr Biol. 26:450-457.

Bakker A, Kirwan CB, Miller M, Stark CEL. 2008. Pattern separation in the human hippocampal CA3 and dentate gyrus. Science (80- ). 319:1640-1642.

Baldassano C, Chen J, Zadbood A, Pillow JW, Hasson U, Norman KA. 2017. Discovering Event Structure in Continuous Narrative Perception and Memory. Neuron. 95:709-721.e5.

Bellmund JL, Deuker L, Doeller CF. 2019. Mapping sequence structure in the human lateral entorhinal cortex. Elife. 8:458133.

Bellmund JLS, Polti I, Doeller CF. 2020. Sequence memory in the hippocampal-entorhinal region. J Cogn Neurosci. 32:2056-2070.

Ben-Yakov A, Dudai Y. 2011. Constructing realistic engrams: poststimulus activity of hippocampus and dorsal striatum predicts subsequent episodic memory. J Neurosci. 31:9032-9042.

Ben-Yakov A, Eshel N, Dudai Y. 2013. Hippocampal immediate poststimulus activity in the encoding of consecutive naturalistic episodes. J Exp Psychol Gen. 142:1255-1263.

Ben-Yakov A, Henson RN. 2018. The Hippocampal Film Editor: Sensitivity and Specificity to Event Boundaries in Continuous Experience. J Neurosci. 38:10057-10068.

Berkers RMWJ, van der Linden M, Neville DA, van Kesteren MTR, Morris RGM, Murre JMJ, Fernandez G. 2018. Neural dynamics of accumulating and updating linguistic knowledge structures. bioRxiv. 495168.

Brewer JB, Zhao Z, Desmond JE, Glover GH, Gabrieli JDE. 1998. Making memories: brain activity that predicts how well visual experience will be remembered. Science (80- ). 281:1185-1187.

Chen J, Honey CJ, Simony E, Arcaro MJ, Norman KA, Hasson U. 2016. Accessing real-life episodic information from minutes versus hours earlier modulates hippocampal and high-order cortical dynamics. Cereb Cortex. 26:3428-3441.

Chen J, Leong YC, Honey CJ, Yong CH, Norman KA, Hasson U. 2017. Shared memories reveal shared structure in neural activity across individuals. Nat Neurosci. 20:115-125.

Cohen JD, Daw N, Engelhardt B, Hasson U, Li K, Niv Y, Norman KA, Pillow J, Ramadge PJ, Turk-Browne NB, Willke TL. 2017. Computational approaches to fMRI analysis. Nat Neurosci. 20:304-313.

Davachi L, DuBrow S. 2015. How the hippocampus preserves order: The role of prediction and context. Trends Cogn Sci. 19:92-99.

Desikan RS, Ségonne F, Fischl B, Quinn BT, Dickerson BC, Blacker D, Buckner RL, Dale AM, Maguire RP, Hyman BT, others. 2006. An automated labeling system for subdividing the human cerebral cortex on MRI scans into gyral based regions of interest. Neuroimage. 31:968-980.

DuBrow S, Davachi L. 2013. The influence of context boundaries on memory for the sequential order of events. J Exp Psychol Gen. 142:1277-1286.

DuBrow S, Davachi L. 2016. Temporal binding within and across events. Neurobiol Learn Mem. 134:107-114.

DuBrow S, Rouhani N, Niv Y, Norman KA. 2017. Does mental context drift or shift? Curr Opin Behav Sci. 17:141146.

Eichenbaum H. 2014. Time cells in the hippocampus: A new dimension for mapping memories. Nat Rev Neurosci. 15:732-744.

Fernández G. 2017. Cognitive Neuroscience of Memory Consolidation.

Fernández G, Effern A, Grunwald T, Pezer N, Lehnertz K, Dümpelmann M, Van Roost D, Elger CE. 1999. Realtime tracking of memory formation in the human rhinal cortex and hippocampus. Science (80- ). 285:15821585.

Genovese CR, Lazar NA, Nichols T. 2002. Thresholding of statistical maps in functional neuroimaging using the 
false discovery rate. Neuroimage. 15:870-878.

Griffiths BJ, Fuentemilla L. 2020. Event conjunction: How the hippocampus integrates episodic memories across event boundaries. Hippocampus. 30:162-171.

Hasson U, Chen J, Honey CJ. 2015. Hierarchical process memory: memory as an integral component of information processing. Trends Cogn Sci. 19:304-313.

Hasson U, Furman O, Clark D, Dudai Y, Davachi L. 2008. Enhanced Intersubject Correlations during Movie Viewing Correlate with Successful Episodic Encoding. Neuron. 57:452-462.

Hasson U, Nir Y, Levy I, Fuhrmann G, Malach R. 2004. Intersubject synchronization of cortical activity during natural vision. Science (80- ). 303:1634-1640.

Hermans EJ, Kanen JW, Tambini A, Fernández G, Davachi L, Phelps EA. 2017. Persistence of amygdala-hippocampal connectivity and multi-voxel correlation structures during awake rest after fear learning predicts long-term expression of fear. Cereb Cortex. 27:3028-3041.

Hermans EJ, Van Marle HJF, Ossewaarde L, Henckens MJAG, Qin S, Van Kesteren MTR, Schoots VC, Cousijn H, Rijpkema M, Oostenveld R, others. 2011. Stress-related noradrenergic activity prompts large-scale neural network reconfiguration. Science (80- ). 334:1151-1153.

Howard MW, Eichenbaum H. 2013. The hippocampus, time, and memory across scales. J Exp Psychol Gen. 142:1211-1230.

Howard MW, Fotedar MS, Datey A V, Hasselmo ME. 2005. The Temporal Context Model in Spatial Navigation and Relational Learning: Toward a Common Explanation of Medial Temporal Lobe Function Across Domains. Psychol Rev. 112:75-116.

Huk A, Bonnen K, He BJ. 2018. Beyond Trial-Based Paradigms: Continuous Behavior, Ongoing Neural Activity, and Natural Stimuli. J Neurosci. 38:7551-7558.

Jafarpour A, Griffin S, Lin JJ, Knight RT. 2019. Medial orbitofrontal cortex, dorsolateral prefrontal cortex, and hippocampus differentially represent the event saliency. J Cogn Neurosci. 31:874-884.

Jenkinson M, Beckmann CF, Behrens TEJ, Woolrich MW, Smith SM. 2012. Fsl. Neuroimage. 62:782-790.

Kim H. 2010. Dissociating the roles of the default-mode, dorsal, and ventral networks in episodic memory retrieval. Neuroimage. 50:1648-1657.

Kim H. 2011. Neural activity that predicts subsequent memory and forgetting: a meta-analysis of 74 fMRI studies. Neuroimage. 54:2446-2461.

Kudrimoti HS, Barnes CA, McNaughton BL. 1999. Reactivation of hippocampal cell assemblies: effects of behavioral state, experience, and EEG dynamics. J Neurosci. 19:4090-4101.

Kumaran D, Summerfield JJ, Hassabis D, Maguire EA. 2009. Tracking the emergence of conceptual knowledge during human decision making. Neuron. 63:889-901.

Kurby CA, Zacks JM. 2018. Preserved neural event segmentation in healthy older adults. Psychol Aging. 33:232245.

Lansink CS, Goltstein PM, Lankelma J V, Joosten RN, McNaughton BL, Pennartz CMA. 2008. Preferential reactivation of motivationally relevant information in the ventral striatum. J Neurosci. 28:6372-6382.

Lositsky O, Chen J, Toker D, Honey CJ, Shvartsman M, Poppenk JL, Hasson U, Norman KA. 2016. Neural pattern change during encoding of a narrative predicts retrospective duration estimates. Elife. 5:1-40.

Manns JR, Howard MW, Eichenbaum H. 2007. Gradual changes in hippocampal activity support remembering the order of events. Neuron. 56:530-540.

Montchal ME, Reagh ZM, Yassa MA. 2019. Precise temporal memories are supported by the lateral entorhinal cortex in humans. Nat Neurosci. 22:284-288.

Nastase SA, Gazzola V, Hasson U, Keysers C. 2019. Measuring shared responses across subjects using intersubject correlation. Soc Cogn Affect Neurosci. 14:667-685.

Paz R, Gelbard-Sagiv H, Mukamel R, Harel M, Malach R, Fried I. 2010. A neural substrate in the human hippocampus for linking successive events. Proc Natl Acad Sci. 107:6046-6051.

Preston AR, Eichenbaum H. 2013. Interplay of Hippocampus and Prefrontal Cortex in Memory. Curr Biol. 23:R764R773.

Qin Y-L, McNaughton BL, Skaggs WE, Barnes CA. 1997. Memory reprocessing in corticocortical and hippocampocortical neuronal ensembles. Philos Trans R Soc London Ser B Biol Sci. 352:1525-1533.

Ranganath C, Ritchey M. 2012. Two cortical systems for memory-guided behaviour. Nat Rev Neurosci. 13:713-726.

Rugg MD, Vilberg KL. 2013. Brain networks underlying episodic memory retrieval. Curr Opin Neurobiol. 23:255260.

Sargent JQ, Zacks JM, Hambrick DZ, Zacks RT, Kurby CA, Bailey HR, Eisenberg ML, Beck TM. 2013. Event segmentation ability uniquely predicts event memory. Cognition. 129:241-255. 
Schaefer A, Kong R, Gordon EM, Laumann TO, Zuo X-N, Holmes AJ, Eickhoff SB, Yeo BTT. 2018. Local-Global Parcellation of the Human Cerebral Cortex from Intrinsic Functional Connectivity MRI. Cereb Cortex. 28:3095-3114.

Schlichting ML, Preston AR. 2015. Memory integration: neural mechanisms and implications for behavior. Curr Opin Behav Sci. 1:1-8.

Schlichting ML, Zeithamova D, Preston AR. 2014. CA1 subfield contributions to memory integration and inference. Hippocampus. 24:1248-1260.

Schwartz MF, Reed ES, Montgomery M, Palmer C, Mayer NH. 1991. The quantitative description of action disorganisation after brain damage: A case study. Cogn Neuropsychol. 8:381-414.

Shirer WR, Ryali S, Rykhlevskaia E, Menon V, Greicius MD. 2012. Decoding subject-driven cognitive states with whole-brain connectivity patterns. Cereb cortex. 22:158-165.

Silva M, Baldassano C, Fuentemilla L. 2019. Rapid Memory Reactivation at Movie Event Boundaries Promotes Episodic Encoding. J Neurosci. 39:8538-8548.

Simony E, Honey CJ, Chen J, Lositsky O, Yeshurun Y, Wiesel A, Hasson U. 2016. Dynamic reconfiguration of the default mode network during narrative comprehension. Nat Commun. 7:12141.

Sols I, DuBrow S, Davachi L, Fuentemilla L. 2017. Event Boundaries Trigger Rapid Memory Reinstatement of the Prior Events to Promote Their Representation in Long-Term Memory. Curr Biol. 27:3499-3504.e4.

Sonkusare S, Breakspear M, Guo C. 2019. Naturalistic Stimuli in Neuroscience: Critically Acclaimed. Trends Cogn Sci. $1-16$.

Spalding KN, Schlichting ML, Zeithamova D, Preston AR, Tranel D, Duff MC, Warren DE. 2018. Ventromedial prefrontal cortex is necessary for normal associative inference and memory integration. J Neurosci. 38:37673775.

Sun C, Yang W, Martin J, Tonegawa S. 2020. Hippocampal neurons represent events as transferable units of experience. Nat Neurosci. 23:651-663.

Tambini A, Davachi L. 2013. Persistence of hippocampal multivoxel patterns into postencoding rest is related to memory. Proc Natl Acad Sci U S A. 110:19591-19596.

Tambini A, Davachi L. 2019. Awake Reactivation of Prior Experiences Consolidates Memories and Biases Cognition. Trends Cogn Sci. 1-15.

Thavabalasingam S, O'Neil EB, Tay J, Nestor A, Lee ACH. 2019. Evidence for the incorporation of temporal duration information in human hippocampal long-term memory sequence representations. Proc Natl Acad Sci. 116:6407-6414.

van Kesteren MTR, Beul SF, Takashima A, Henson RN, Ruiter DJ, Fernández G. 2013. Differential roles for medial prefrontal and medial temporal cortices in schema-dependent encoding: from congruent to incongruent. Neuropsychologia. 51:2352-2359.

van Kesteren MTR, Rijpkema M, Ruiter DJ, Fernández G. 2010. Retrieval of associative information congruent with prior knowledge is related to increased medial prefrontal activity and connectivity. J Neurosci. 30:1588815894.

van Kesteren MTR, Rijpkema M, Ruiter DJ, Morris RGM, Fernández G. 2014. Building on prior knowledge: schema-dependent encoding processes relate to academic performance. J Cogn Neurosci. 26:2250-2261.

Wagner AD, Schacter DL, Rotte M, Koutstaal W, Maril A, Dale AM, Rosen BR, Buckner RL. 1998. Building memories: remembering and forgetting of verbal experiences as predicted by brain activity. Science (80- ). 281:1188-1191.

Xue G. 2018. The neural representations underlying human episodic memory. Trends Cogn Sci. 22:544-561.

Yassa MA, Stark CEL. 2011. Pattern separation in the hippocampus. Trends Neurosci. 34:515-525.

Zacks JM. 2020. Event Perception and Memory. Annu Rev Psychol. 71:165-191.

Zacks JM, Speer NK, Swallow KM, Braver TS, Reynolds JR. 2007. Event perception: a mind-brain perspective. Psychol Bull. 133:273.

Zacks JM, Tversky B, Iyer G. 2001. Perceiving, remembering, and communicating structure in events. J Exp Psychol Gen. 130:29-58.

Zeithamova D, Dominick AL, Preston AR. 2012. Hippocampal and ventral medial prefrontal activation during retrieval-mediated learning supports novel inference. Neuron. 75:168-179. 